\title{
A fully coupled computational fluid dynamics - agent-based model of atherosclerotic plaque development: multiscale modeling framework and parameter sensitivity analysis
}

\author{
Anna Corti ${ }^{1}$, Claudio Chiastra ${ }^{1,2}$, Monika Colombo' ${ }^{1}$, Marc Garbey ${ }^{3,4,5}$, Francesco \\ Migliavacca1, Stefano Casarin $^{3,4,6}$
}

1. Laboratory of Biological Structure Mechanics (LaBS), Department of Chemistry, Materials and Chemical Engineering "Giulio Natta”, Politecnico di Milano, Milan, Italy

2. PoliTo ${ }^{\mathrm{BIO}} \mathrm{Med} \mathrm{Lab}$, Department of Mechanical and Aerospace Engineering, Politecnico di Torino, Turin, Italy

3. Center for Computational Surgery, Houston Methodist Research Institute, Houston, TX, USA

4. Department of Surgery, Houston Methodist Hospital, Houston TX, USA

5. LASIE UMR 7356 CNRS, University of La Rochelle, La Rochelle, France

6. Institute for Academic Medicine (IAM), Houston, TX, USA

Address for correspondence:

Dr. Claudio Chiastra, PhD

PoliTo ${ }^{\mathrm{BIO}}$ Med Lab

Department of Mechanical and Aerospace Engineering

Politecnico di Torino

Corso Duca degli Abruzzi, 24

10129 Turin, Italy

E-mail: claudio.chiastra@polito.it

ORCID: http://orcid.org/0000-0003-2070-6142 


\begin{abstract}
Background. Peripheral artery disease (PAD) is an atherosclerotic disorder that leads to unpaired lumen patency through intimal hyperplasia and the build-up of plaques, mainly localized in areas of disturbed flow. Computational models can provide valuable insights in the pathogenesis of atherosclerosis and act as a predictive tool to optimize current interventional techniques. Our hypothesis is that a reliable predictive model must include the atherosclerosis development history. Accordingly, we developed a multiscale modeling framework of atherosclerosis that replicates the hemodynamic-driven arterial wall remodeling and plaque formation.
\end{abstract}

Methods. The framework was based on the coupling of Computational Fluid Dynamics (CFD) simulations with an Agent-Based Model (ABM). The CFD simulation computed the hemodynamics in a 3D artery model, while 2D ABMs simulated cell, extracellular matrix (ECM) and lipid dynamics in multiple vessel cross-sections. A sensitivity analysis was also performed to evaluate the oscillation of the ABM output to variations in the inputs and to identify the most influencing ABM parameters.

Results. Our multiscale model qualitatively replicated both the physiologic and pathologic arterial configuration, capturing histological-like features. The ABM outputs were mostly driven by cell and ECM dynamics, largely affecting the lumen area. A subset of parameters was found to affect the final lipid core size, without influencing cell/ECM or lumen area trends.

Conclusion. The fully coupled CFD-ABM framework described atherosclerotic morphological and compositional changes triggered by a disturbed hemodynamics.

Keywords: Atherosclerosis, computer modeling, multiscale model, agent-based model, lipid plaque, SMC, ECM, remodeling, wall shear stress, hemodynamics 


\section{Introduction}

Peripheral Arterial Disease (PAD) is an atherosclerosis-related pathology characterized by arterial lumen stenosis through the formation of a lipid-rich plaque, which affects more than 200 million people worldwide [1]. In its severe stage, the disease can potentially lead to peripheral gangrene, with consequent amputation, or spread to other parts of the body exposing the patient to the risk of heart attack or stroke [2].

Within the multifactorial nature of the pathology, the relation between hemodynamics and atherosclerotic plaque localization has been extensively studied, supported by evidences of plaque occurrence at sites of disturbed blood flow, where a low Wall Shear Stress (WSS) is appreciable [3]. The role of WSS in the pathogenesis of atherosclerosis has been associated with induced mechanotransduction at cellular level. WSS modulates the endothelial release of specific chemicals that control pivotal states such as the permeability to low density lipoproteins and cellular/extracellular functions, including Smooth Muscle Cells (SMCs) proliferation and Extracellular Matrix (ECM) dynamics, which are all relevant within the disease pathogenesis [4].

Nowadays, the preferred techniques adopted to restore the physiological circulation, namely Percutaneous Transluminal Angioplasty (PTA) and Vein Graft Bypass (VGB), are still affected by a high rate of medium/long-term failure, with a 3-years patency of $60 \%$ for PTA and a 5-years patency of $70 \%$ for VGB [5]. An interdisciplinary approach and advances in the study of the underlying cellular, molecular and genetic mechanisms involved in atherosclerosis are widening the actual knowledge of the disease and will facilitate the improvement of the current therapeutic solutions and development of new strategies $[6,7]$.

Mathematical models and computational simulations are powerful tools that can provide great support and guidance in this research process [8,9]. A variety of computational models have been used 
to investigate the clinical outcome of PTA with or without stenting [10-12] and VGB confirming the prominent need to overcome the limitations associated with these procedures, e.g. [13,14]. Particular attention was directed to the investigation of the in-stent restenosis mechanisms, a maladaptive vascular response to PTA and stent deployment which, leading to lumen re-occlusion, is considered one of the main causes of failure of the treatment [15]. In this context, several research groups used Agent-Based models (ABMs) to replicate the restenotic vascular remodeling processes following PTA with stenting, exalting the potentialities of $\mathrm{ABM}$ in simulating the response of a biological system to the alteration of its baseline working conditions [16-24]. However, most of the aforementioned works introduced the perturbation, namely the intervention procedure, in a healthy artery, without considering the underlying pathology. In the study by Curtin and Zhao [24], a more realistic pathological geometry was adopted as initial configuration and the atherosclerotic plaque was modeled, even though as an inert entity. Our hypothesis is that a more reliable predictive model of intervention should include the pathological history that, besides modifying the geometry and composition of the arterial wall, leads to an alteration of the cellular and extracellular dynamics and the activation of processes, for example due to the presence of the lipid core, that might affect the treatment outcome. To the best of our knowledge, few works adopted ABM techniques to simulate atherosclerotic plaque formation and their main focus was on the immune and inflammatory events occurring in the early phases of atherosclerosis [25-27].

Accordingly, we here present a multiscale framework that, starting from an idealized healthy artery, simulates the process of atherosclerotic plaque formation and progression generating a diseased artery as output. This is an extension of a previous preliminary work from our research group [28] where we coupled Computational Fluid Dynamics (CFD) simulations with agent-based modeling to replicate hemodynamic-driven events in atherosclerosis. Specifically, the CFD analysis performed in a 3D artery model provides the hemodynamic input to multiple 2D ABMs, which, in turn, simulate cellular dynamics. In [28] we provided a preliminary description of the working mechanisms, which will be 
here discussed in detail. Moreover, additional work was done to improve the model reliability, with peculiar attention directed to the sensitivity analysis of the ABM, performed to evaluate the model output in response to variations at the level of the input parameters. Indeed, due to the lack of a direct calibration of the driving coefficients of the model on experimental data, those coefficients were selected heuristically, leading to a certain level of uncertainty that needed to be quantified. The sensitivity analysis allowed us to understand the impact of the uncertain inputs on the model response, identifying the most influencing parameters, whose future calibration on experimental data will improve the model accuracy [29]. Finally, the results of this analysis provided further understandings of the model mechanisms, namely a verification of the ABM response with respect to the model laws, and the identification of an unexpected or not considered behavior.

\section{Material and methods}

\subsection{Multiscale framework}

Figure 1 shows the structure of the complete multiscale framework that consists of four cyclically repeated steps [28]. First, an idealized 3D model of the lumen of a tortuous portion of healthy Superficial Femoral Artery (SFA) is built and a 3D mesh of the fluid dynamic domain is generated using ICEM CFD (v. 18.0, Ansys Inc., Canonsburg, PA, USA). To compute the hemodynamics, a steady-state CFD simulation is performed in Fluent (v. 18.0, Ansys Inc.), and the WSS profiles are extracted at the lumen interface of a discrete number $(M=10)$ of $2 \mathrm{D}$ vessel cross-sections. For each plane, the local, hemodynamic-driven remodeling is replicated with an ABM implemented in Matlab (v. 2016b, MathWorks, Natick, MA, USA) that simulates cellular, extracellular and lipid dynamics. Specifically, depending on the WSS profile computed by the CFD simulation, the ABM replicates the physiologic or pathologic arterial wall remodeling occurring in a predefined time-frame (e.g. one week 
$\mathrm{ABM}$ simulated time). At the end of said period, here referred as coupling period, the ABM simulations are stopped to perform a new CFD simulation in the modified (i.e. updated) 3D geometry. Indeed, the geometrical changes computed by the ABM in each $2 \mathrm{D}$ plane affect the fluid dynamic domain, implying the need to update the hemodynamics and the WSS distribution by coupling back the ABMs to the CFD module. To do that, a new 3D geometry of the lumen vessel is reconstructed by lofting the luminal curves of the $M$ ABM outputs and the four main steps of the multiscale framework are then re-performed. The entire process stops at the end of a predefined follow-up period (e.g. at two months ABM simulated time). Each of the aforementioned steps, as well as the coupling process, require the user intervention.

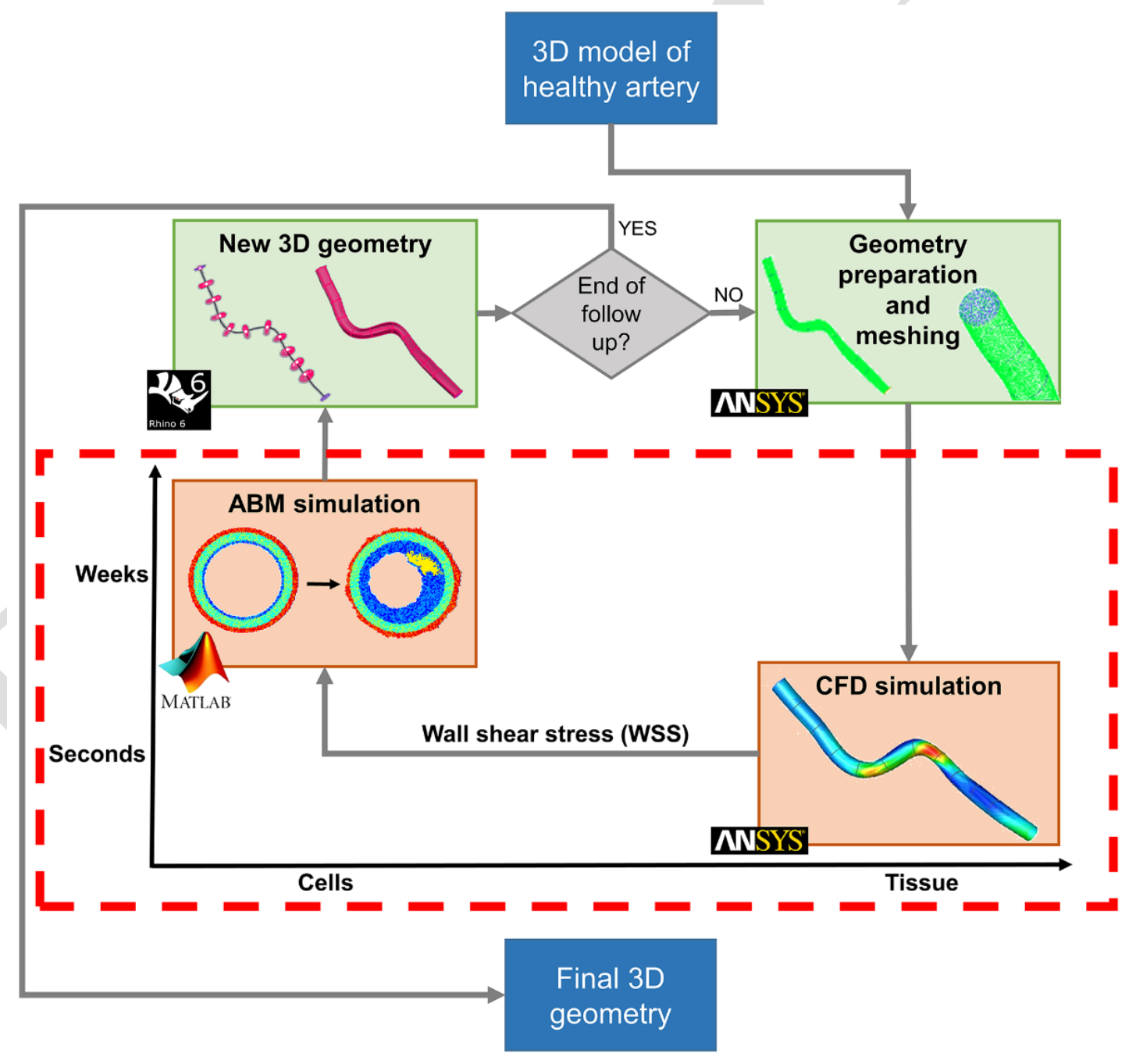


Fig. 1. Multiscale computational framework [28]. Starting from a 3D model of healthy artery, the physiologic/pathologic wall remodeling is simulated through a four-blocks scheme: (i) geometry preparation and meshing, (ii) CFD simulation, (iii) $\mathrm{ABM}$ simulation and (iv) new 3D geometry. The CFD and $\mathrm{ABM}$ modules constitute the multiscale core of the framework, acting on the second/tissue and weeks/cell scales, respectively.

The multiscale core is based on the CFD and the ABM modules, embedded in the dashed red box in Fig. 1. The first simulates at tissue/seconds scale the average hemodynamics in the SFA, while the second replicates at cell scale the process of arterial wall remodeling, involving a time span of weeks/months.

A step-by-step extended description is provided below with the following order: i) geometry preparation and meshing, ii) CFD simulation, iii) ABM simulation, and iv) retrieval of the new 3D geometry.

\subsubsection{Geometry preparation and meshing}

A simplified 3D geometry of the lumen of a healthy artery with SFA-like features was initially built using the CAD software Rhinoceros (v. 6.0, Robert McNeel \& Associates, Seattle, WA, USA) (Fig. 2A). The initial geometry presented a centerline length of $L_{c e n t}=84.02 \mathrm{~mm}$, and circular inlet and outlet cross-sections with diameters $D_{\text {inlet }}=4.10 \mathrm{~mm}$ and $D_{\text {outlet }}=3.71 \mathrm{~mm}$, respectively.

The 3D geometry was imported into ICEM CFD to generate the 3D mesh of the lumen for the CFD simulation. A hybrid tetrahedral mesh with five boundary layers of prism elements was created with the Octree method [30]. As global mesh parameters, an element maximum size of $0.39 \mathrm{~mm}$ was set and the curvature/proximity based refinement was enabled with a minimum element size of $0.156 \mathrm{~mm}$ and a refinement of 20 edges along a radius of curvature. The five layers of prism elements were generated with an exponential growth law, setting 1.05 as height ratio. Finally, the mesh was globally smoothed 
by imposing five smoothing iterations and a quality criterion up to 0.4 . The resulting mesh, shown in Fig. 2B, presented almost 235500 cells.

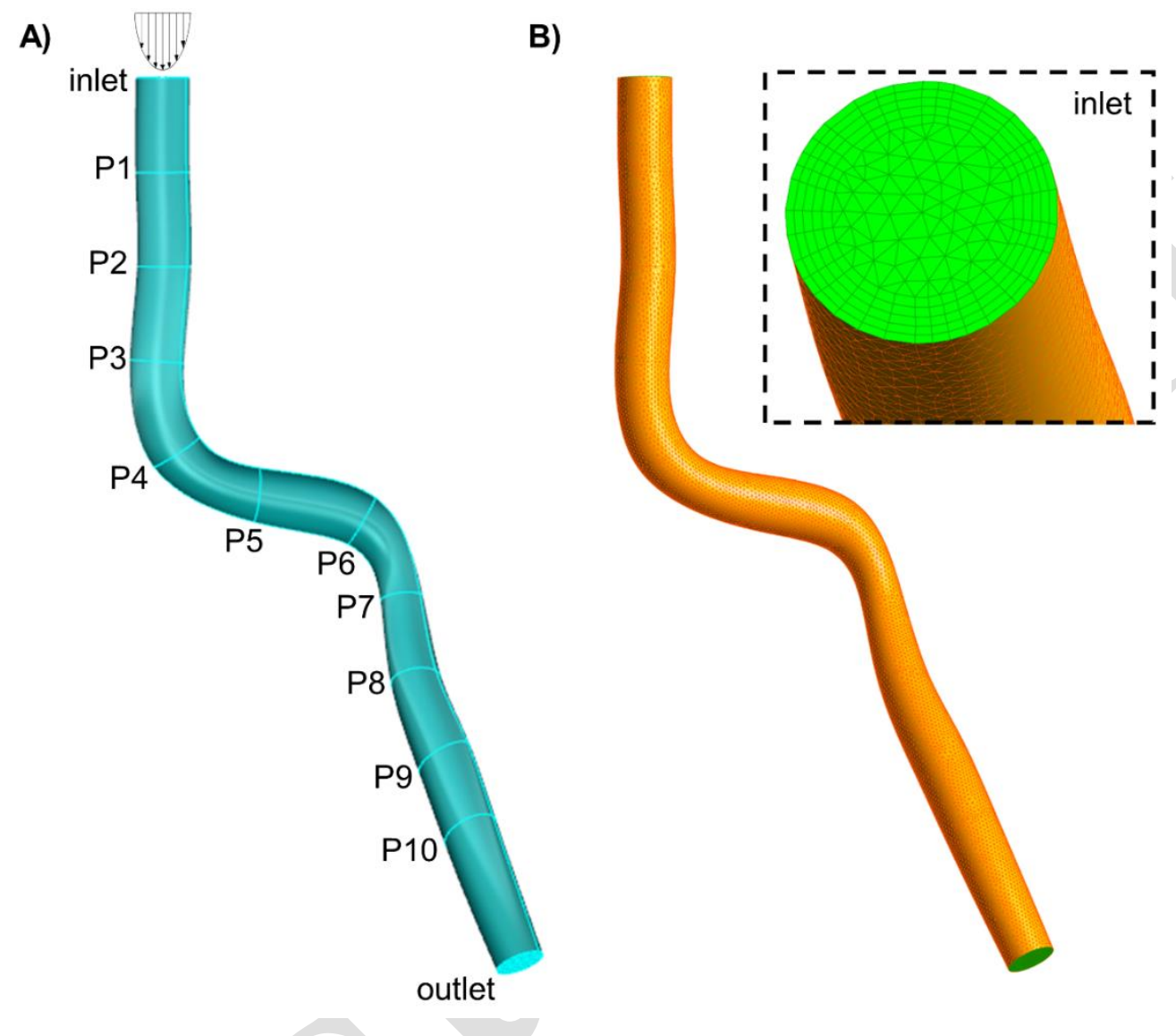

Fig. 2. Fluid dynamic domain. A) 3D ideal geometry of the superficial femoral artery, with ten cross-sectional planes P1-10 where the wall shear stress profiles were extracted as hemodynamic input for the agent-based model (ABM). B) Mesh of the fluid domain, with a magnified view of the inlet cross-section, showing the tetrahedral mesh and five layers of prism elements next to the arterial lumen.

\subsubsection{CFD simulation}

Steady-state CFD simulations were performed using Fluent to compute the hemodynamics in the 3D artery model. Since the arterial wall remodeling, computed by the ABM, occurs in the time scale of weeks, while the cardiac output waveform is in the order of the seconds, cellular dynamics were assumed to depend on the average WSS. Accordingly, to avoid excessive time consumption, a steady flow was imposed to approximate the average hemodynamics. 
At the inlet cross-section, a constant parabolic-shaped velocity profile with physiological mean velocity was applied. The mean velocity was derived from the analysis of patient's Doppler ultrasound image at the SFA level [31], following a proper scaling to make it consistent with the current inlet area. At the outlet cross-section, a reference zero pressure was imposed. No-slip wall boundary condition was specified at the arterial lumen, assumed as rigid. A density of $\rho=1060 \mathrm{~kg} / \mathrm{m}^{3}$ was set for blood, modeled as a non-Newtonian Carreau fluid, as in [32]. The simulation was run using the pressure-based solver with coupled method as pressure-velocity coupling method, least square cell-based scheme for the spatial discretization of the gradient, second-order scheme for the pressure and second-order upwind scheme for the momentum spatial discretization [33].

At the end of the CFD simulation, WSS profiles were extracted at pre-selected $M=10$ internal circular planes perpendicular to the centerline (Fig. 2A) and used as hemodynamic input to the corresponding ABM. The choice of $M=10$ planes guaranteed a reliable reconstruction of the $3 \mathrm{D}$ vessel geometry.

\subsubsection{ABM simulation}

In general, starting from an initial homeostatic condition, the 2D ABM replicates a physiologic or pathologic arterial wall remodeling (depending on the WSS profile) on a vessel wall cross-section, by locally simulating cell mitosis/apoptosis, ECM production/degradation and lipid infiltration in the intima. The model was developed assuming that the risk factors promoting the disease were already present. Accordingly, the process of plaque formation in a specific region is purely driven by the hemodynamic conditions, and specifically by the WSS profile.

The implemented ABM was inspired to the one developed by Garbey et al. [34,35] for the simulation of VGB post-surgical adaptation. However, different vessel structure and composition, as 
well as agent types and dynamics were implemented in the present work, which also deals with new cellular events.

Figure 3 shows the ABM flowchart. Following the geometrical and hemodynamic initialization, at each time step of one hour, the model computes cell/ECM and lipid dynamics that drive the remodeling of the wall. Then, in order to retrieve smooth profiles and guarantee structural integrity, the lumen and external walls are regularized at each iteration until the end of the simulated period.

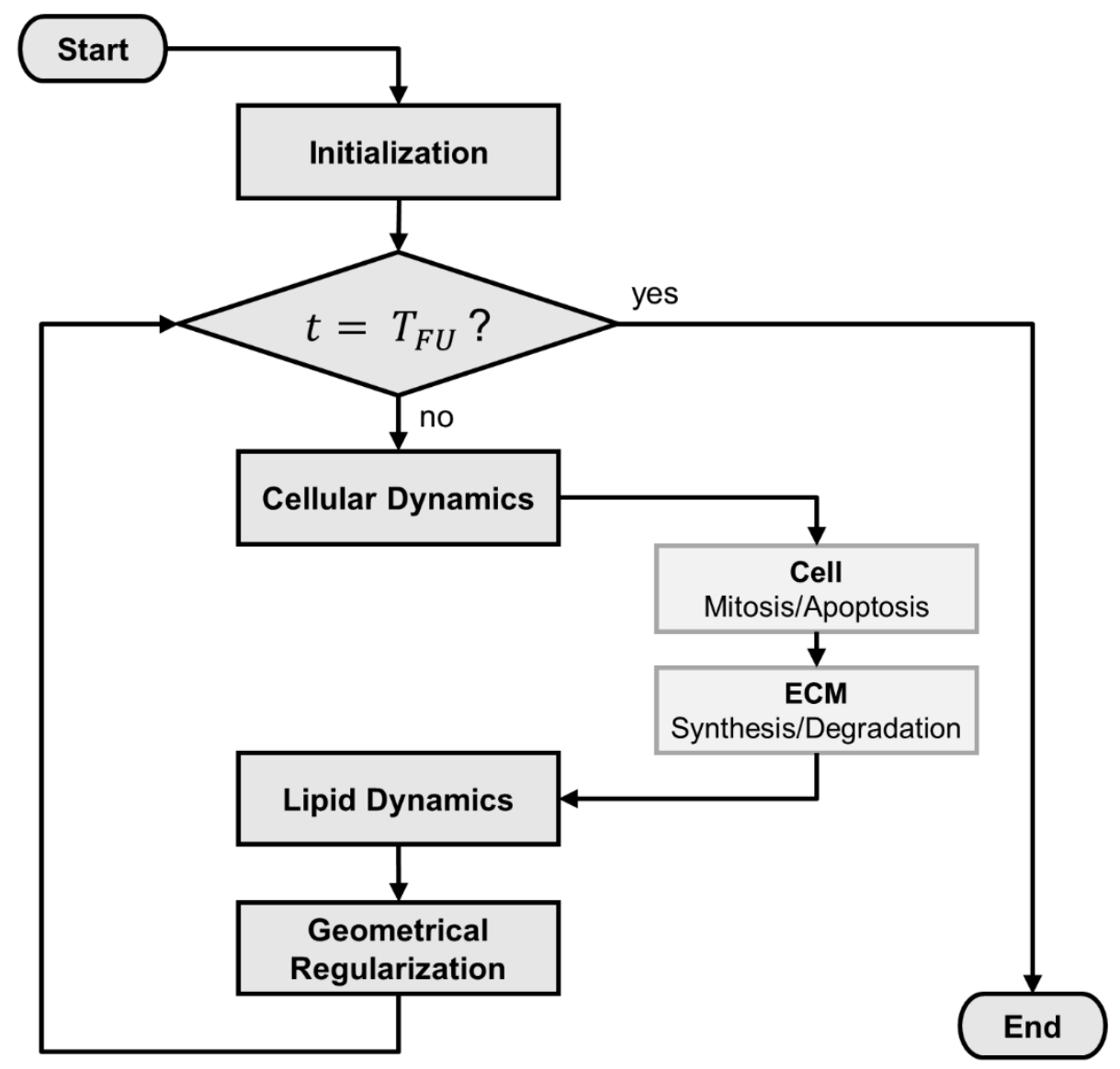

Fig. 3. Agent-based model $(\mathrm{ABM})$ flowchart. $t$ refers to the internal clock of the $\mathrm{ABM}$ and $\mathrm{T}_{\mathrm{FU}}$ is the predefined followup time.

To replicate the cellular and extracellular events, probabilistic behavioral rules were assigned to each agent and the simulations were performed with Monte Carlo method, allowing capturing the intrinsic variability of biological processes. Due to the stochasticity of the present ABM, the output of a single simulation cannot be considered as a representative solution. Thus, $N=10$ independent 
simulations were run starting from the same initial condition and the average trend was evaluated. The choice of $N$ was dictated by the need of a reasonable trade-off between computational time and minimization of the standard deviation.

A basic solution was generated by opportunely calibrating the agent dynamics in order to stabilize the system around an equilibrium working point. This condition, representative of the homeostatic state of a healthy artery, was then perturbed to simulate the process of atherosclerotic plaque formation.

Prior to the building of the fully coupled framework, the ABM behavior was verified both under physiologic and atherogenic conditions. For this purpose, a single CFD-ABM coupling was performed to initialize the ABM with the hemodynamic input and the ABM simulations were run for two months. Differently, within the fully coupled framework, the ABM running time corresponded to the chosen coupling time between ABM and CFD modules.

The ABM simulations were run on a 16.00 GB RAM CPU, Intel ${ }^{\circledR}$ Core $^{\mathrm{TM}}$ i7-4790, with 4 Cores and 8 Logical Processors.

Details on the ABM initialization, agent dynamics and geometrical regularization are provided below.

Initialization. The ABM was implemented on a 2D $<130 \times 130>$ hexagonal grid, representing a good compromise between affinity to the isotropic reality and level of complexity and computational efforts. The initial geometry is a $2 \mathrm{D}$ circular cross-section composed by 3 concentric layers, i.e. tunica intima, media and adventitia (Fig. 4A) with the internal and external elastic laminae (IEL and EEL) separating the intima and the media and the media and adventitia, respectively (Fig. 4B). While sites in the lumen and in the external portion to the wall are initially empty, each site within the wall is randomly seeded with a cell or an ECM, coherently with the cell/ECM ratio of each layer [36-38]. Intima and media are initialized with SMCs and elastin and collagen as ECM, with a SMC/ECM ratio 
of 0.72 [36] and a collagen/elastin ratio of 0.63 [38], while fibroblasts and collagen fill the adventitial sites with a ratio of 0.43 [37]. Figures 4C and 4D show cellular and extracellular composition of intima, media and adventitia layers. For simplicity, no differentiated behaviors between SMC and fibroblasts or between elastin and collagen were implemented.

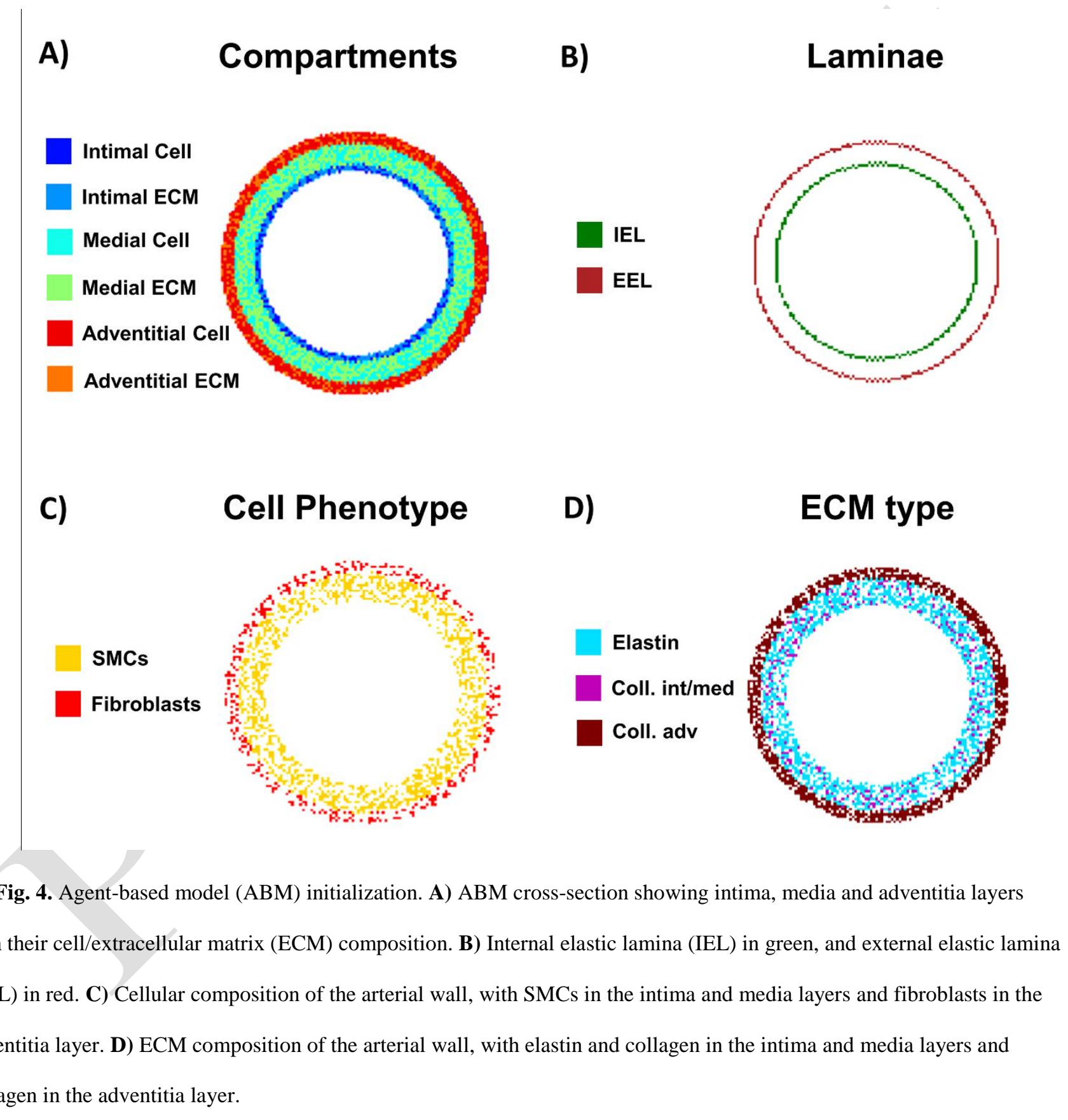


As previously mentioned, the 2D ABM is informed with an initial WSS profile, which can potentially trigger a pathologic vascular remodeling by perturbing the baseline cellular activity and favoring lipid infiltration and accumulation within the arterial wall $[3,4]$. Indeed, a low WSS affects the endothelial function by down-regulating atheroprotective genes and up-regulating the atherogenic ones, eventually promoting atherosclerotic plaque formation $[4,39]$.

Accordingly, each site $i$ of the lumen wall is initialized with a WSS value obtained from the 3D CFD simulation, and a level of endothelial dysfunction $D^{i}$ is computed as follows:

$$
D(W S S)^{i}=D^{i}=\left\{\begin{array}{c}
1-\frac{W S S^{i}}{W S S_{0}}, \text { if } W S S^{i}<W S S_{0} \\
0, \text { otherwise }
\end{array}\right.
$$

$W S S^{i}$ is the WSS at site $i$ and $W S S_{0}=1 P a$ is the assumed pathologic/physiologic WSS threshold. $W S S_{0}$ was set in accordance with the study of Samady et al. [40], in which areas exposed to WSS lower than $1 \mathrm{~Pa}$ developed greater lumen area reduction. Moreover, the chosen threshold agrees with the physiological range of WSS in the SFA, identified to be between 1.5-2 $\mathrm{Pa}$ [41].

In the ABM, each dysfunctional endothelial site $i$, with $D^{i} \neq 0$, triggers a state of alteration that diffuses within the intima through isotropic diffusion, from a peak of intensity $D^{i}$ with a diffusion constant $\phi$ :

$$
A^{i, k}\left(D^{i}, d\right)=A^{i, k}=D^{i} * e^{-\frac{1}{2}\left(\frac{d}{4 \phi t}\right)^{2}}
$$

where $A^{i, k}\left(D^{i}, d\right)$ is the level of alteration generated by the $i$-th endothelial site and recorded at the $k$-th site within intima, at a distance $d$ from $i$. In each site $k$, the individual states of alteration originated 
from different endothelial sites are summed up to define the global level of inflammation of the $k$-th site $I^{k}$, as follows:

$$
I^{k}=\sum_{i=1}^{N_{L}} A^{i, k}
$$

$N_{L}$ is the initial number of sites of the lumen wall (i.e. endothelial sites) and the resulting $I^{k}$ affects the agent dynamics, as described below, promoting atherosclerotic plaque formation.

Since the purpose of the present model was not to accurately replicate the mechanisms of endothelial dysfunction and the early inflammatory processes occurring during atherogenesis, the endothelium and inflammatory cells or molecules were not explicitly modeled. However, Eqs (1) - (3) were implemented to capture the key role of the hemodynamic input in the pathogenesis of atherosclerosis, whose effect, thanks to the mediation of the endothelial layer, is transferred to the interior sites. Specifically, if all the WSS values at the $i$-th sites are greater than the threshold, $D^{i}=$ $0 \forall i$ and $I^{k}=0$ everywhere. Under this condition, defined atheroprotective, the homeostasis of a healthy artery is replicated. On the contrary, if there is at least one site of the lumen wall exposed to a $W S S^{i}<W S S_{0}$, a state of inflammation $I$ develops and the mechanisms of plaque formation are activated, namely the lipid dynamics and an increased cellular activity in the intima. The related WSS profile was thus defined atherogenic.

The geometrical and compositional initialization shown in Fig. 4 and described in the present section is performed only at the first cycle of the framework in Fig. 1. The starting geometrical and compositional configurations of the ABM simulations at the cycle $n+1$ are the corresponding $\mathrm{ABM}$ output configuration of the previous cycle, $n$, which are informed with the respective WSS profile obtained from the CFD simulations of the current cycle, $n+1$. 
Agent dynamics. The ABM at $\mathrm{t}=0$ is populated with cells and ECM, for which each component is approximated as a single agent. To desynchronize the cellular activity and ensure the stochastic nature of the simulation, an internal clock was associated to the model. Each site containing a cell/ECM was initially associated with a random number, $t_{\text {agent }}$, that individuates its biological state within the relative cycle, of period $T_{\text {site }}$, which was differentially defined for cells, $T_{\text {site }}=T_{\text {cell }}=12$ hours, and $\mathrm{ECM}, T_{\text {site }}=T_{\text {matrix }}=2$ hours. $t_{\text {agent }}$ is incremented of one hour at each time step and an agent can potentially undergo a certain event (described later) only when $t_{\text {agent }}=n * T_{\text {site }}$, with $n$ positive integer number.

When a site containing cell/ECM is accessed (i.e. when it is in its potentially active state), a Monte Carlo simulation determines whether the potential event is happening or not, as shown in the "event assessment" phase of Fig. S1. The CPU generates a random number test $\in[0 ; 1]$ that is compared with the probability of the event itself, $p_{\text {event }}$, and the event occurs if test $<p_{\text {event }}$.

The cellular events of interest are, as baseline of activity, mitosis/apoptosis for cells and deposition/degradation for ECM, and, only under atherogenic condition, the ABM also simulates the process of lipids infiltration.

In Fig. 5, the workflow adopted for the implementation of the agent dynamics and the corresponding parameter setting is shown. A detailed analysis of the biological processes occurring during atherosclerosis initiation and progression was performed, with a focus on the cellular, extracellular and lipid dynamics, which led to the definition of their probabilistic behavioral rules. The final probability equations depend on a set of coefficients $\alpha_{i}$, adopted to weigh a specific influencing factor in the global agent behavior or to set the probability in the interval $(0: 1)$.

The numerical value assumed by those coefficients was derived through an iterative process in which the output of the ABM was verified in terms of integrity and qualitative resemblance to histological or literature evidences. In the present section, the values obtained from the aforementioned 
process are listed below each equation and tagged as default values. This procedure allowed us to obtain a reasonable range for each coefficient. However, since they were not experimentally derived, they are associated with uncertainty, which is reflected in the model output. For this purpose, a sensitivity analysis of the $\alpha_{i}$ parameters was performed and detailed in section 2.2.1. In future works, the parameters $\alpha_{\mathrm{i}}$ that emerged as driving ABM coefficients will be calibrated against experimental data and the ABM will be finally validated.

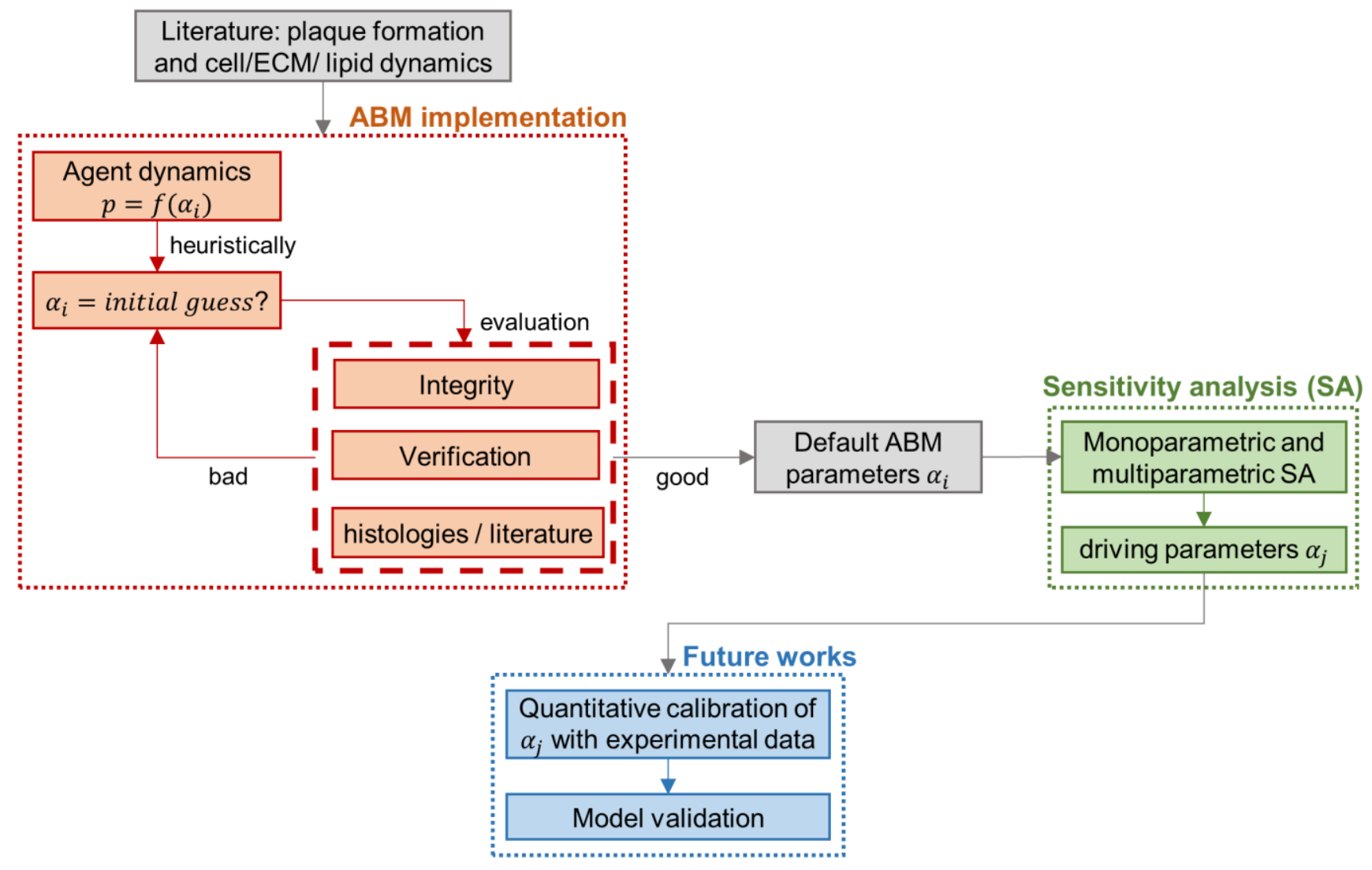

Fig. 5. Workflow for the implementation and fine-tuning of the agent-based model (ABM).

Before implementing the pathological cellular dynamics, the baseline densities of probability were set for cell mitosis/apoptosis and ECM deposition/degradation to replicate the physiological conditions. They were defined with Eq. (4) and Eq. (5), respectively: 


$$
\begin{aligned}
& p_{\text {mit }}=p_{\text {apop }}=\alpha_{1} \\
& p_{\text {prod }}=\beta * p_{\text {deg }}=\alpha_{4}
\end{aligned}
$$

$\alpha_{1}, \alpha_{4}$ and $\beta$ were imposed to guarantee the maintenance of the physiologic cell/ECM ratio defined at the initialization phase for each tissue layer. $\alpha_{1}$ and $\alpha_{4}$ were set to compensate the different cellular and extracellular time period, i.e. $\alpha_{1}=\frac{0.1}{T_{\text {matrix }}}=0.05$ and $\alpha_{4}=\frac{0.1}{T_{\text {cell }}}=0.008$, where, the 0.1 multiplier was introduced to obtain a baseline probability of said event within a realistic unit of measure [34].

While cell agents are responsible for cell mitosis and apoptosis and ECM production, ECM agents are involved in ECM degradation, meaning that the code scans the grid looking for cells or ECM, respectively. It results that, due to the prevalence of ECM on cells, the model has the tendency to preferentially degrade ECM, instead of producing it. Accordingly, to replicate a baseline condition where ECM production and degradation are averagely balanced, an adjusting coefficient $\beta$ was introduced and calibrated for each layer. Specifically, since the intima and the media layer have the same cell/ECM ratio $=0.72$, a single $\beta_{\text {int/med }}$ was defined for these two layers, while a different value, $\beta_{\text {adv }}$, for the adventitia, being the adventitia composed by a cell/ECM ratio of 0.43 . To this aim, ten ABM simulations were run under physiologic conditions with several tentative $\beta_{\text {int/med }}$ and $\beta_{\text {adv }}$ values and, at a 2-months follow-up, the ratio between final and initial ECM, $\frac{E C M_{f}}{E C M_{i}}$, was computed for each layer. Considering the work of Garbey et al. [34], a first set of ten simulations was run with initial guesses of $\beta_{\mathrm{int} / \mathrm{med}}=2.13$ and $\beta_{\mathrm{adv}}=2.5$ and provided $\frac{E C M_{f}}{E C M_{i}}>1$ in the intima and media layers and $\frac{E C M_{f}}{E C M_{i}}=1$ in the adventitia layer. Other five values corresponding to $1,1.5,1.6,1.75$ and 2 were investigated to calibrate $\beta_{\mathrm{int} / \mathrm{med}}$ and, by interpolating the $\frac{E C M_{f}}{E C M_{i}}$ vs. $\beta$ plot in correspondence of $\frac{E C M_{f}}{E C M_{i}}=$ $1, \beta_{\text {int } / \text { med }}=1.57$ was identified as adjusting coefficient for the intima and the media layers, as shown in 
Fig. 6. Therefore, $\beta=\{1.57,1.57,2.5\}$ were set for intima, media and adventitia, respectively, in order to guarantee stable trends of ECM in each layer under baseline conditions.

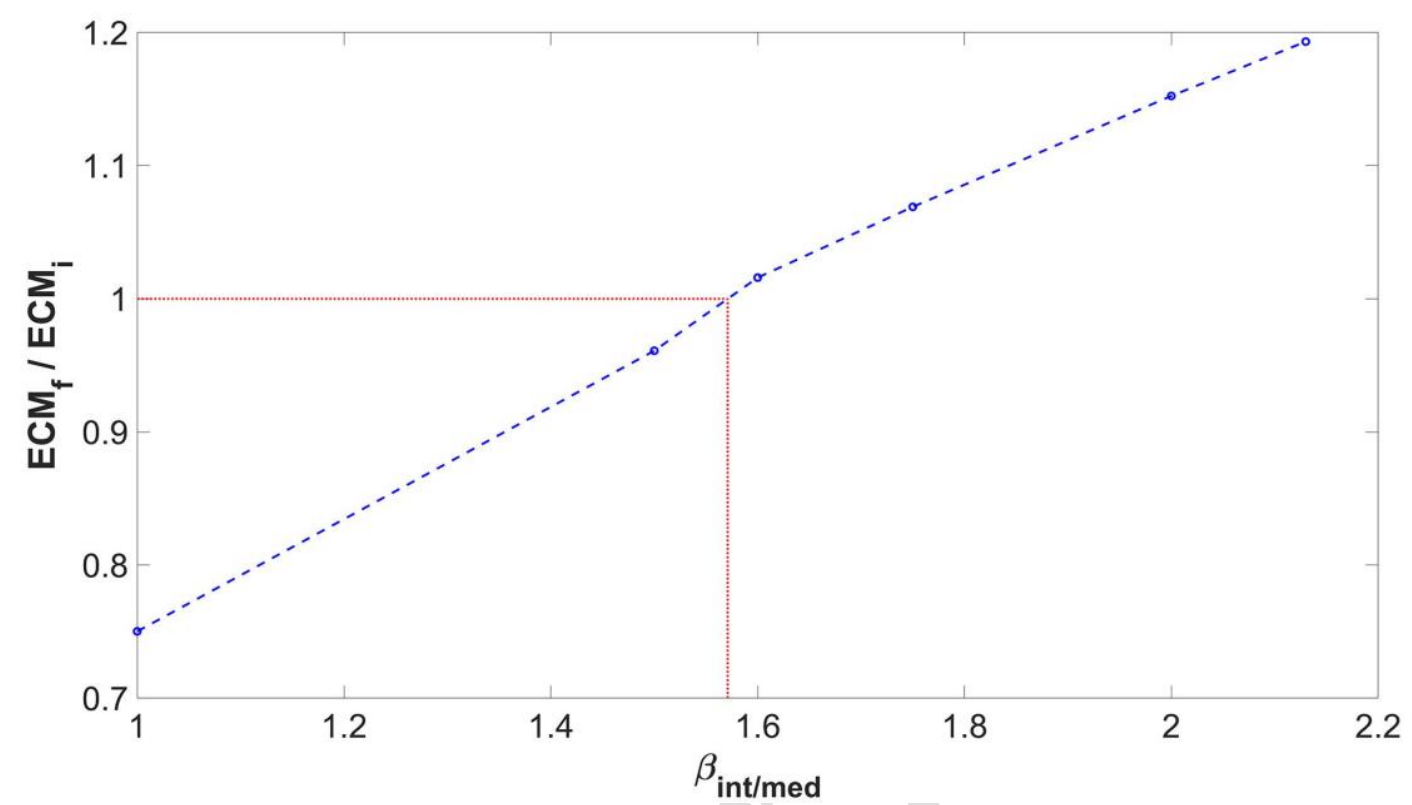

Fig. 6. $\beta_{\text {int } / \text { med }}$ interpolation. The $\frac{E C M_{f}}{E C M_{i}}$ values obtained at different $\beta_{\text {int } / \text { med }}=1,1.5,1.6,1.75,2,2.13$ with fixed $\beta_{\text {adv }}=2.5$ are indicated as blue circles. Each of the $\frac{E C M_{f}}{E C M_{i}}$ values is the average result of five repeated simulations. The dashed red curve indicates the interpolation of $\beta_{\text {int/med }}$ in correspondence of $\frac{E C M_{f}}{E C M_{i}}=1$.

With said calibrated coefficients, Eq. (4) and Eq. (5) drive the physiological wall remodeling, leading to the replication of the homeostatic state of a healthy artery. Differently, under atherogenic conditions (i.e. when at least one site of the lumen wall is associated with a WSS $<1 P a$ ) cellular mitosis and ECM production in the intima are perturbed to model the increased cellular activity involving the intima layer during atherosclerosis. This translates into a modification of the probability densities defined with Eq. (4) and Eq. (5). Specifically, the probability of cell mitosis and ECM production in the intima increases with the inflammation level, the number of neighboring lipids and the closeness to the lumen [42], leading to the following: 


$$
\begin{aligned}
& p_{\text {mit }}=\left\{\begin{array}{c}
\alpha_{1} \cdot\left(1+\alpha_{2} I^{k}\right) \text { if } n_{\text {lip }}=0 \\
\alpha_{1} \cdot\left(1+\alpha_{2} I^{k}\right)\left(1+\alpha_{3} n_{\text {lip }}\right)\left\{1+\exp \left(-d_{\text {lumen }}^{k}\right)\right\} \text { if } n_{\text {lip }} \neq 0
\end{array}\right. \\
& p_{\text {prod }}=\left\{\begin{array}{c}
\alpha_{4} \cdot\left(1+\alpha_{2} I^{k}\right) \text { if } n_{\text {lip }}=0 \\
\alpha_{4} \cdot\left(1+\alpha_{2} I^{k}\right)\left(1+\alpha_{3} n_{\text {lip }}\right)\left\{1+\exp \left(- \text { dist }_{\text {lumen }}^{k}\right)\right\} \text { if } n_{\text {lip }} \neq 0
\end{array}\right.
\end{aligned}
$$

$\alpha_{2}=1.5$ and $\alpha_{3}=0.1$ weigh, respectively, the effect of the inflammation state, $I^{k}$, and the influence of the neighboring lipids, $n_{\text {lip }}$, while $d_{\text {lumen }}^{k}$ is the distance between the site $k$ and the lumen wall. The coefficients were set following the framework proposed in Fig. 5, with the additional requisites of obtaining probability values of the agent dynamics in the interval $(0 ; 1)$ and an accelerated plaque formation under atherogenic condition, so that the pathological processes in the planes exposed to atherogenic WSS profile arose within two simulated months. This choice, although not realistic, was dictated by the need to reduce the elevated computational time.

Under atherogenic conditions, the ABM also implements the process of lipid infiltration in the intima. In order to simulate an earlier adaptive intimal thickening [3], lipid dynamics is activated once the intima thickens over a given threshold, here set as $I T=6$ sites. Since circulating low density lipoproteins were not explicitly modeled, the probability of lipid infiltration is computed as the probability of a site $k$ at the lumen wall to allow lipids to invade the intima, expressed by:

$$
p_{\text {lipid }}=\alpha_{5}\left(1+I^{k}\right)\left\{1+\alpha_{6} \cdot \exp \left(-d i s t_{\text {lip }}^{k}\right)\right\}\left(1+\frac{n_{\text {lip }}}{\alpha_{7}}\right)
$$

where $\alpha_{5}=0.05$ sets the event probability in the interval $(0 ; 1)$. The terms $\alpha_{6} \exp \left(-d i s t_{\text {lip }}^{k}\right)$ and $\left(1+\frac{n_{\text {lip }}}{\alpha_{7}}\right)$ promote lipid clustering, by increasing the probability of a lipid to occupy a site $k$ close to 
another lipid, whose distance is $d i s t_{\text {lip }}^{k}$ and whose neighboring lipids is, in turn, $n_{\text {lip }} . \alpha_{6}=10$ weighs the distance term between $k$ and its closest lipid, and $\alpha_{7}=6$ is a normalization constant to maintain the ratio in the interval $(0 ; 1)$. Also in this case, the terms and coefficients of Eq. (8) were set following the framework in Fig. 5 to obtain a lipid core resembling histological features [43].

At each time step only one lipid can enter the intima. To determine the site of access for the lipid, the ten sites of the lumen wall with higher $p_{\text {lipid }}$ are explored. Starting from the most probable site Monte Carlo simulation is applied and if $p_{\text {lipid }}^{k}>$ test, then $k$ is the designated site of access, otherwise the following site of the list is investigated, up to ten. This translates in assuming that a lipid has a total number of chances, $\operatorname{tr} y_{\text {lip }}=10$, to migrate into the intima.

In the present work, it was assumed that lipids might continue entering the intima until the lipid core potentially occupies maximum $15 \%$ of the lumen area. Although not representative of real biological mechanisms, this condition allowed replicating a progressive growth followed by a stabilization of the lipid core, which agrees with the choice not to simulate the phenomenon of plaque rupture, more likely associated with a continuous growth of the lipid-rich necrotic core [44]. Moreover, in PAD, which is the context of the current study, atherosclerotic plaques are usually characterized by a smaller lipid core than in coronary artery disease, and are less subjected to rupture, which further corroborates the assumption on the lipid core size [43].

Tissue plasticity and geometrical regularization. To accommodate the production or removal of an element while performing agent dynamics (“event manifestation” phase of Fig. S1), the tissue reorganizes by following a minimum energy principle, according to which agents move along the shortest path to the target site [34]. In case the active site is at the luminal or external wall border, the production of a cell/ECM results in the addition of a new agent, positioned in a random empty space surrounding the active agent itself. In same condition, if the active agent undergoes death or 
degradation, it is simply removed from the computational domain leaving an empty space. Differently, in case of an agent inside the arterial wall, a pushing or pulling movement of the surrounding elements allows the production or removal of an element. For example, in case of element production, a site adjacent to the mitotic/synthetic cell is freed thanks to the movement of the surrounding elements either towards the lumen or the exterior, respectively if the site is in the intima or in the media/adventitia. Similarly, when an element is removed, its site is occupied by an inverse movement of the neighboring agents. Agent movement must always comply with the minimum energy principle, with the only exception constituted by the presence of lipid agents along the shortest path. Once they enter the intima layer, lipid agents must maintain their position throughout the entire simulation, thus constituting an obstacle to the movement of the surrounding elements. Consequently, the agent movement is performed along the shortest path that does not involve lipid agents, allowing preserving the lipid core.

Figure 7 provides, on a magnified schematic portion of the arterial wall (Fig. 7A), an example of possible rearrangements of the tissue in case of production or removal of an agent in the intima (Fig. 7B), media (Fig. 7C) and adventitia layers (Fig. 7D). 
A.

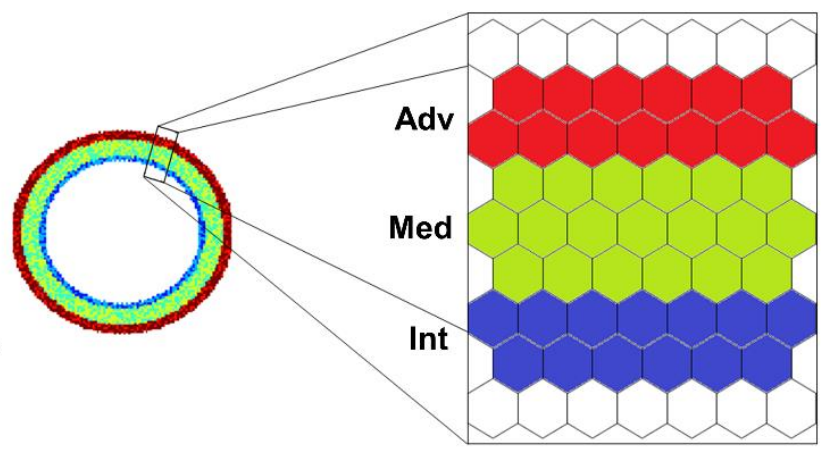

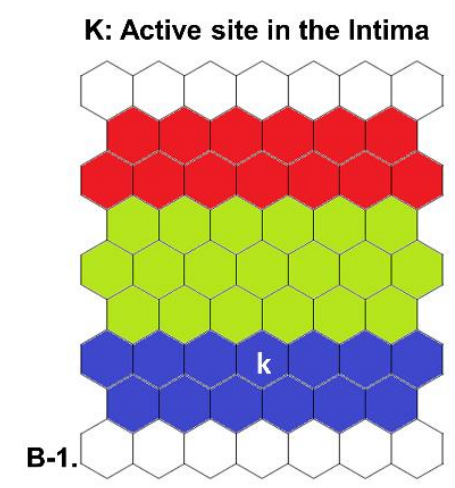

K: Active site in the Media

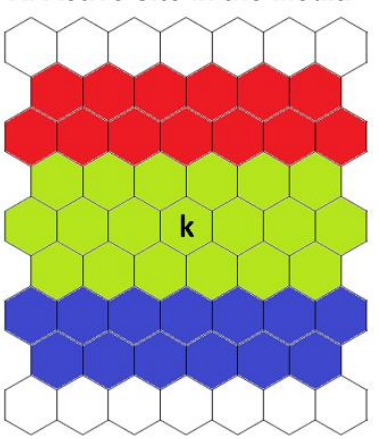

C-1.

K: Active site in the Adventitia

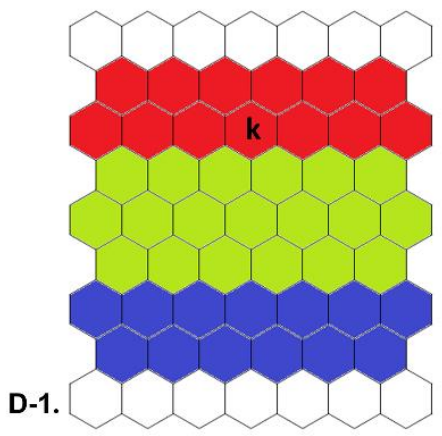

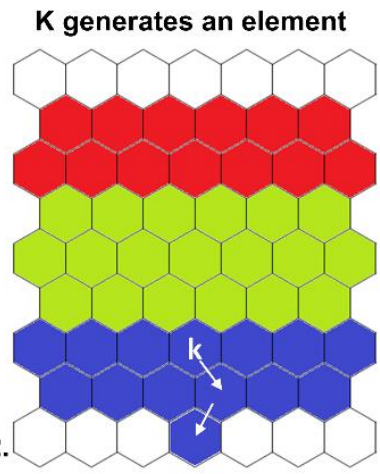

$\mathrm{K}$ generates an element

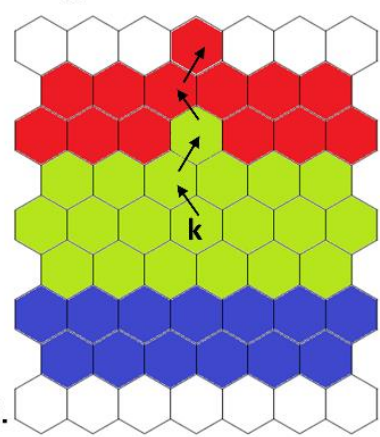

$K$ generates an element

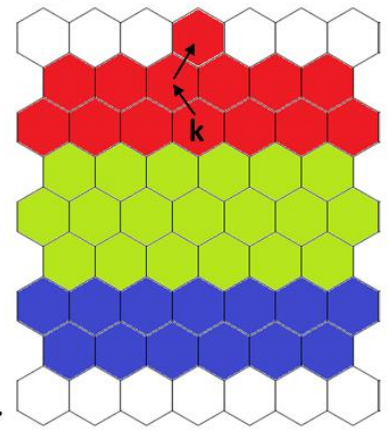

B-3.

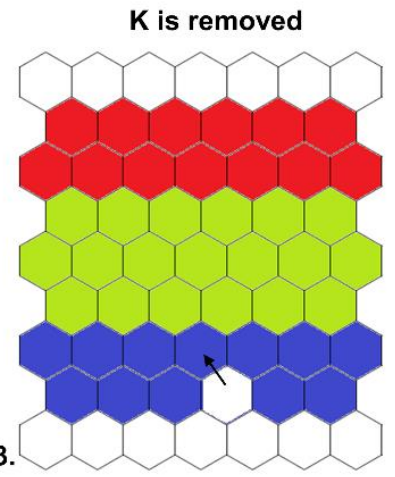

C-3.

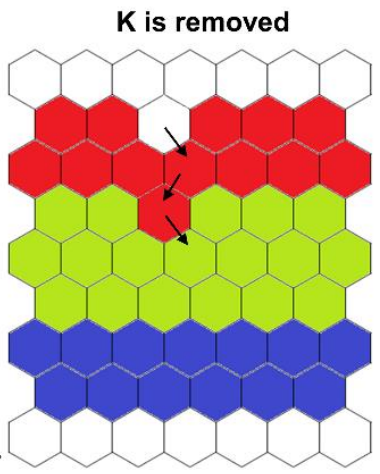

$\mathrm{K}$ is removed

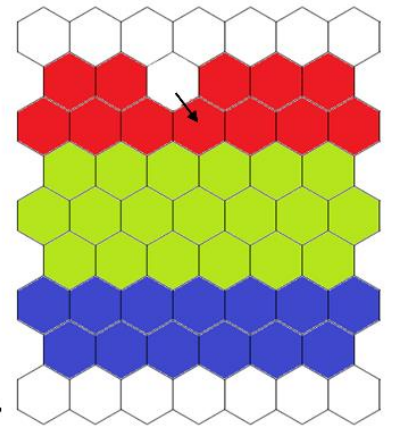

Fig. 7. Examples of tissue rearrangement. A) Schematic representation of a magnified portion of the arterial wall, with the intima in blue, the media in green and the adventitia in red. Examples of tissue reorganization when the active site $\mathrm{k}$ produces an element or is removed in the intima (B), media (C) and adventitia (D). 
Finally, in order to guarantee integrity of the structure and smooth profiles at the interface, a regularization is applied at each time step. The regularization involves the luminal wall, the internal elastic lamina, the external elastic lamina and the exterior border with different algorithms depending on the case. The luminal and external boundaries are regularized through a continuous movement of the border sites until the achievement of a compact structure, which maximizes contacts among agents by redistributing potential spikes. The internal and external elastic laminae are instead adjusted by computing their average radii and performing target agent movements to obtain regular separations among the tissue layers while preserving the layer composition and area resulting from the computation of the agent dynamics. The regularization step, besides allowing obtaining a realistic structure, is of crucial importance to guarantee the correct functioning of the model, preventing potential degeneration and instabilities.

\subsubsection{Retrieval of the new 3D geometry}

Within the fully coupled framework, at the end of the ABM simulation period, $N=10$ output solutions, different in terms of morphology, composition and plaque features, are obtained for each of the $M=10$ cross-sections of the artery. Accordingly, an innovative method was developed to select for each cross-section the output configuration that mostly resembled the corresponding average solution in terms of i) lumen radius, ii) external radius, and iii) plaque size. The procedure, described below, allowed building, at the end of each cycle of the framework in Fig. 1, a unique 3D geometry of the lumen vessel, and determining the starting ABM configuration of each plane for the following cycle of the framework. Specifically, for each ABM solution, $\mathrm{i}=\{1, \ldots, N\}$, of a given $M$ cross-section, lumen and external radii and plaque thickness as function of the angular coordinate $\vartheta$ were computed and indicated as $R_{j}^{i}(\vartheta)$, with $\mathrm{j}=1,2,3$, respectively. The corresponding deviation, $\Delta^{i}$, from the average 
configuration, $\overline{R_{J}(\vartheta)}$, was computed as defined in Eq. (9), and the ABM $i$-th output minimizing $\Delta$ was selected.

$$
\Delta^{i}=\sum_{j=1}^{3} \int_{0}^{2 \pi} w_{j} \sqrt{\left(R_{j}^{i}(\vartheta)-\overline{R_{\jmath}(\vartheta)}\right)^{2}} d \vartheta
$$

where each $j$-th quantity is weighed by $w_{j}$.

The same criterion was applied for all the $M=10$ cross-sections and the $3 \mathrm{D}$ geometry was finally reconstructed in Rhinoceros by lofting the lumen profiles of the selected configurations.

\subsection{Sensitivity analysis}

Sensitivity analyses of the ABM coefficients and of the ABM/CFD coupling period were performed and detailed below in sections 2.2.1 and 2.2.2, respectively. In the first analysis, the response of the 2D $\mathrm{ABM}$ was studied at the variation of its driving parameters with the aims of gaining further insights on the $\mathrm{ABM}$ working mechanisms and identifying, if present, the dominant parameters. In turn, the analysis of the coupling time was carried out on the fully coupled framework to investigate the sensitivity of the scheme to different frequencies of update of the hemodynamic condition, with the final goal of determining a suitable coupling period that limits the computational efforts without affecting the results.

\subsubsection{ABM sensitivity analysis}

Since the model output is largely affected by the parameter setting and none of the parameters was derived from experiments, a sensitivity analysis of the ABM parameters was performed. The goals were (i) to evaluate the oscillation of the model solution due to the uncertainty of the parameters or to the possible inter-subjects variability and (ii) to identify the parameters that mainly drive the ABM output. 
For this purpose, first a mono-parametric and then a multi-parametric sensitivity analyses were carried out, as detailed below. In both analyses we defined $\mathrm{v}=\left\{\alpha_{2}, \alpha_{3}, \alpha_{5}, \alpha_{6}, \alpha_{7}, I T\right.$, try lip $\}$ that consists in the parameter set under investigation. $\alpha_{1}$ and $\alpha_{4}$ were not included in the analysis because they were already calibrated in [34]. For each parameter, a triangular probability density function was defined, based on the parameter range and its most probable value, as shown in Tab. 1. The parameter range was chosen to be consistent with the physical meaning of the parameter itself, while guaranteeing agent behavioral probabilities between 0 and 1 when said parameter is varied and all the others are maintained at their default values. About the most likely value, the default value defined in the previous section was considered for each parameter.

Tab. 1 Default value and range of the parameters for the sensitivity analysis

\begin{tabular}{ccc}
\hline Parameter & Baseline value & Range \\
\hline$\alpha_{2}$ & 1.5 & $(2 ; 17)$ \\
$\alpha_{3}$ & 0.1 & $(0 ; 0.5)$ \\
$\alpha_{5}$ & 0.005 & $(0 ; 0.106)$ \\
$\alpha_{6}$ & 10 & $(0 ; 24.46)$ \\
$\alpha_{7}$ & 6 & $(1.84 ; 100)$ \\
IT $_{\text {try }}$ & 6 & $(2 ; 17)$ \\
& 10 & {$[1 ; 20]$} \\
\hline
\end{tabular}

Aligned with the purpose of the current analysis, all the ABM simulations were initialized with the same WSS profile, corresponding to the one computed at the $9^{\text {th }}$ plane of the SFA-like geometry (Fig. 2A). Indeed, being the most critical hemodynamic scenario, it activates a prompt and intense atherogenic response, allowing appreciating the effects of parameter variation in the $\mathrm{ABM}$ response 
within just one month of follow-up. This is convenient, considering the high computational costs required by the sensitivity analysis. Moreover, since the focus of the analysis was the ABM, the hemodynamic update was not considered, but only 2D ABM simulations were run.

Mono-parametric sensitivity analysis. The probability density function of each parameter was divided in five equal probability intervals and the medium value for each interval was considered. Moreover, two additional values were included in the analysis to explore the ABM behavior at the extremes of the parameter range, thus investigating seven values for each of the seven parameters, shown in Tab. 2. As mentioned above, in this analysis only one parameter at a time was varied, while keeping all the others at their default values, resulting in 49 cases, each with ten replicates.

The results were analyzed in terms of lumen area and intimal content of SMCs, ECM and lipids. Indeed, the intima is the layer that is mostly affected by the pathologic wall remodeling occurring in atherosclerosis.

The statistical analysis of the results was performed in Matlab. The normality of data was assessed using Kolmogorov-Smirnov test. After verifying that all data were not parametric, Kruskal-Wallis test with multiple comparison was used to detect differences among the variables. Tukey-Kramer method was applied for the $\mathrm{p}$-value corrections, and variables were considered significantly different if $\mathrm{p}$-value $<0.05$.

Tab 2. Parameter samples for mono-parametric sensitivity analysis

\begin{tabular}{cccccccc}
\hline Parameter & Value 1 & Value 2 & Value 3 & Value 4 & Value 5 & Value 6 & Value 7 \\
\hline $\boldsymbol{\alpha}_{\mathbf{2}}$ & 0.029 & 0.465 & 1.123 & 1.468 & 1.805 & 2.436 & 2.854 \\
$\boldsymbol{\alpha}_{3}$ & 0.005 & 0.050 & 0.127 & 0.185 & 0.259 & 0.400 & 0.495 \\
$\boldsymbol{\alpha}_{5}$ & 0.001 & 0.016 & 0.039 & 0.052 & 0.064 & 0.089 & 0.105 \\
$\boldsymbol{\alpha}_{6}$ & 0.245 & 3.488 & 8.428 & 11.215 & 14.290 & 20.244 & 24.215 \\
$\boldsymbol{\alpha}_{7}$ & 2.822 & 7.935 & 19.781 & 32.352 & 48.053 & 78.467 & 99.018
\end{tabular}




\begin{tabular}{cccccccc} 
IT & 2.150 & 3.728 & 6.249 & 7.954 & 10.054 & 14.121 & 16.850 \\
try $_{\text {lip }}$ & 1 & 3 & 8 & 10 & 12 & 17 & 20 \\
\hline
\end{tabular}

Multi-parametric sensitivity analysis. Latin hypercube sampling (LHS) was adopted to randomly sample the triangular probability density function of each parameter and define the parameter set for the ABM simulations [45]. This method allows exploring the entire range of each parameter and achieves good accuracy with a limited number of simulations compared to simple random sampling [45]. In this study, the probability density functions of the $j=7\left\{\alpha_{2}, \alpha_{3}, \alpha_{5}, \alpha_{6}, \alpha_{7}, I T\right.$, try $\left.y_{l i p}\right\}$ parameters were divided into $k=10$ equal probability intervals and the LHS matrix $(k \mathrm{x} j)$ was generated, identifying the $k=10 \mathrm{ABM}$ parameter combinations:

$$
\text { LHS matrix }=\left[\begin{array}{ccccccc}
2.308 & 0.211 & 0.074 & 11.901 & 14.616 & 12.799 & 18 \\
1.004 & 0.339 & 0.066 & 7.710 & 50.609 & 7.039 & 1 \\
1.597 & 0.154 & 0.049 & 19.369 & 24.559 & 3.829 & 9 \\
0.915 & 0.359 & 0.036 & 1.102 & 6.838 & 4.452 & 13 \\
2.004 & 0.124 & 0.102 & 9.159 & 42.310 & 8.682 & 11 \\
1.421 & 0.228 & 0.031 & 10.211 & 64.241 & 10.368 & 8 \\
0.395 & 0.054 & 0.062 & 5.937 & 70.664 & 15.746 & 12 \\
1.731 & 0.096 & 0.014 & 15.608 & 12.999 & 5.812 & 6 \\
1.287 & 0.148 & 0.041 & 18.063 & 28.455 & 9.035 & 10 \\
1.940 & 0.260 & 0.052 & 14.116 & 34.063 & 7.463 & 15
\end{array}\right]
$$

For each $k$-th parameter set, ten simulations were run to account for the inherent stochasticity. Partial Rank Correlation Coefficients (PRCC) were computed to quantify the correlation of the target outputs (i.e. lumen area and intimal content of SMCs, ECM and lipids) with each parameter, while removing the effect of the remaining parameters. To compute the PRCC, the average target outputs of the ten replicates for each $k$-th simulation was considered [45]. PRCC can span from -1 to +1 , corresponding to a perfect negative/positive correlation, respectively, and a p-value is associated to each correlation to assess the statistical significance. Correlations were considered statistically significant if the corresponding p-value was lower than 0.05 . 


\subsubsection{Sensitivity analysis of the coupling time}

Back to the fully coupled CFD-ABM framework, an important decisional step was about the definition of the coupling time, namely at which time step the ABM simulations need to be paused to update the hemodynamics according to the geometrical changes. A short coupling period allows a better control of the model, but implies high computational time and efforts. Accordingly, a compromise between accuracy of the results and computational effort must be reached. To this aim, we developed an innovative technique based on a sensitivity analysis that was performed to assess the influence of the coupling time on the output, by testing three different cases on a 14 days follow-up period. In the first two cases, the ABM was coupled back to the CFD with a frequency of 7 and 3.5 days, respectively, while, in the third case, the first coupling was performed after 7 days, and then every 3.5 days. For each cross-section, the temporal evolution of the lumen area predicted in the three cases was evaluated, as well as the ABM simulation mode at each coupling interval, which can be either physiologic or pathologic, depending on the WSS profile computed at the corresponding coupling step.

\section{Results}

\subsection{ABM replication of homeostasis and atherosclerotic plaque generation}

The ABM accurately and robustly replicated both the homeostatic condition of a healthy artery and the formation of an atherosclerotic plaque, when subjected to the hemodynamic stimuli.

Figure 8 shows a qualitative comparison between histology and the ABM output, with the latter selected among the $N$ independent runs for plane 1 (Fig. 8A) and plane 5 (Fig. 8B), for visualization purposes. When initialized with a physiologic WSS profile, the ABM output on a 2-months simulation did not show any substantial deviation from the initial configuration, as depicted in Fig. 8A. The slight 
alteration of the wall profile was within the physiological range, guaranteeing the preservation of the lumen and tissue layers areas. On the contrary, under atherogenic conditions, the ABM developed an atherosclerotic lesion with features resembling histological evidences, as shown in Fig. 8B. Both the ABM atherosclerotic output and the corresponding histology presented an asymmetric geometry, due to a focally localized thickening of the intima layer and the formation of a lipid-rich core (Fig. 8B). However, in the ABM output (Fig. 8B) the thick layer of fibrous intimal tissue covering the lipid core was not present because lipids were still migrating in the intima at the stage of the ABM configuration in Fig. 8B. By implementation, such layer would form once the process of lipid infiltration arrests and SMCs and ECM remain the only active agents, coherently with the fact that lipid core formation precedes the increase of fibrous tissue [46]. However, in the present work, the tissue layer separating the lipid core from the lumen was not fibrotic but normal intima, namely SMCs and ECM. Finally, in the ABM solution, maintenance of baseline thickness and composition of the media and adventitia layer was in good agreement with the histological image. 
A)

Healthy artery

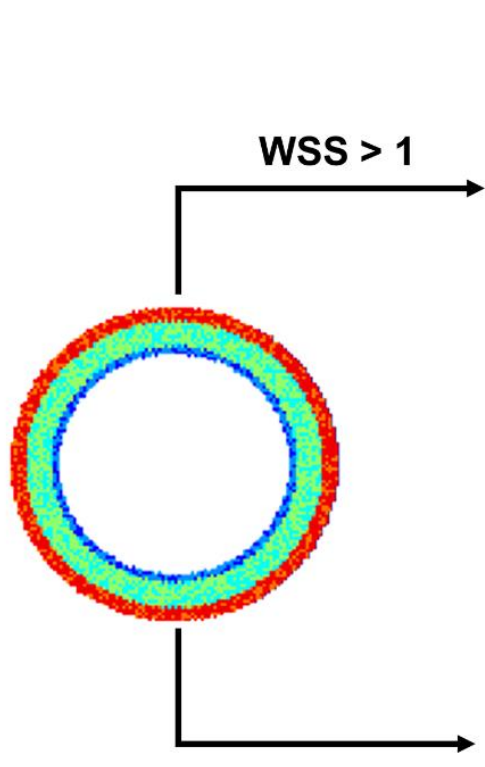

WSS $<1$
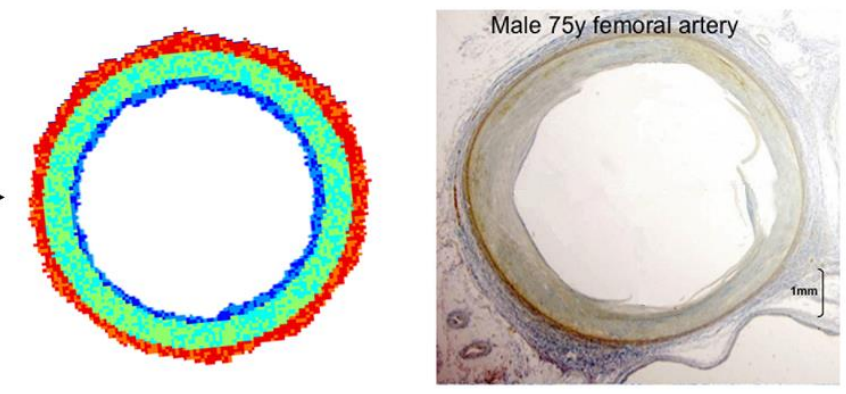

B) Atherosclerotic artery
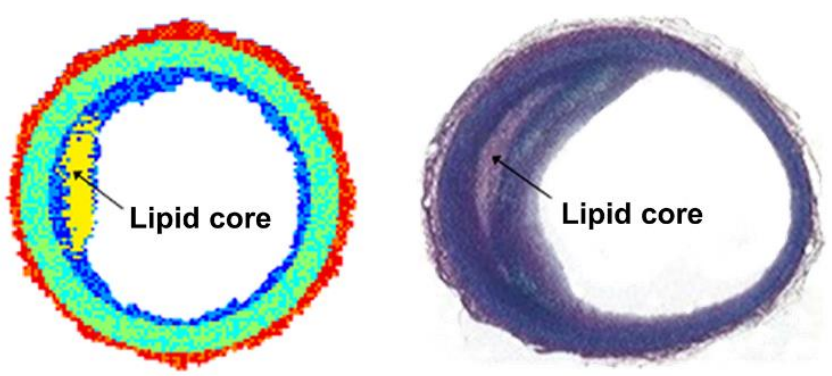

Fig. 8. Comparison between the 2-months agent-based model (ABM) outputs and histological images when the $A B M$ is initialized with a physiologic (A) or pathologic (B) WSS profile. One ABM cross-section out of ten is shown for plane 1 (A) and plane 5 (B), as example of physiologic and atherogenic hemodynamic condition, respectively. The histology in (A) refers to a femoral artery of a 75-years old male subject [47], while in (B) to a coronary fibrous cap atheroma of a 24-years old man [48].

For each plane, the analysis of the temporal evolution of the ABM simulations and outputs allowed a further evaluation of the model dynamics and robustness, both in physiologic and pathologic mode. Under physiologic condition, stable trends of total cells, ECM and wall area were observed, and final healthy configurations were generated (Fig. S2). As shown in Figs. S2-3, although the variability attributable to the inherent stochasticity, a good agreement among the outputs was appreciable and indicative of a robust replication of homeostasis.

Focusing on the atherogenic condition, further results are provided for the vessel cross-sectional plane 5 in Fig. 9. However, similar considerations apply for all the other cross-sections showing plaque 
formation. In Fig. 9, the results of the ten ABM simulations of arterial wall remodeling of plane 5 on a 2-months follow-up are illustrated in terms of temporal dynamics (Fig. 9A) and final ABM configurations (Fig. 9B). The normalized temporal trends of intimal, lumen, medial and adventitial area are provided, pointing out the monotonic decrease in lumen area due to the intimal thickening and the stability of media and adventitia layers. A considerable variation among the simulations involved the most active dynamics, i.e. the luminal and intimal areas, while little to negligible deviation was observed in adventitial and medial areas, respectively (Fig. 9A). The ABM output solutions at 2 months are provided in Fig. 9B for each of the a,...,j simulations. All the configurations replicated a pathologic wall remodeling with plaque generation, the latter shown in yellow. Although the intrinsic variability among the outputs due to the stochastic nature of the model, all the solutions agreed in terms of degree of stenosis and plaque size, location and morphology, as well as unaltered media and adventitia. 
A)

Temporal trends
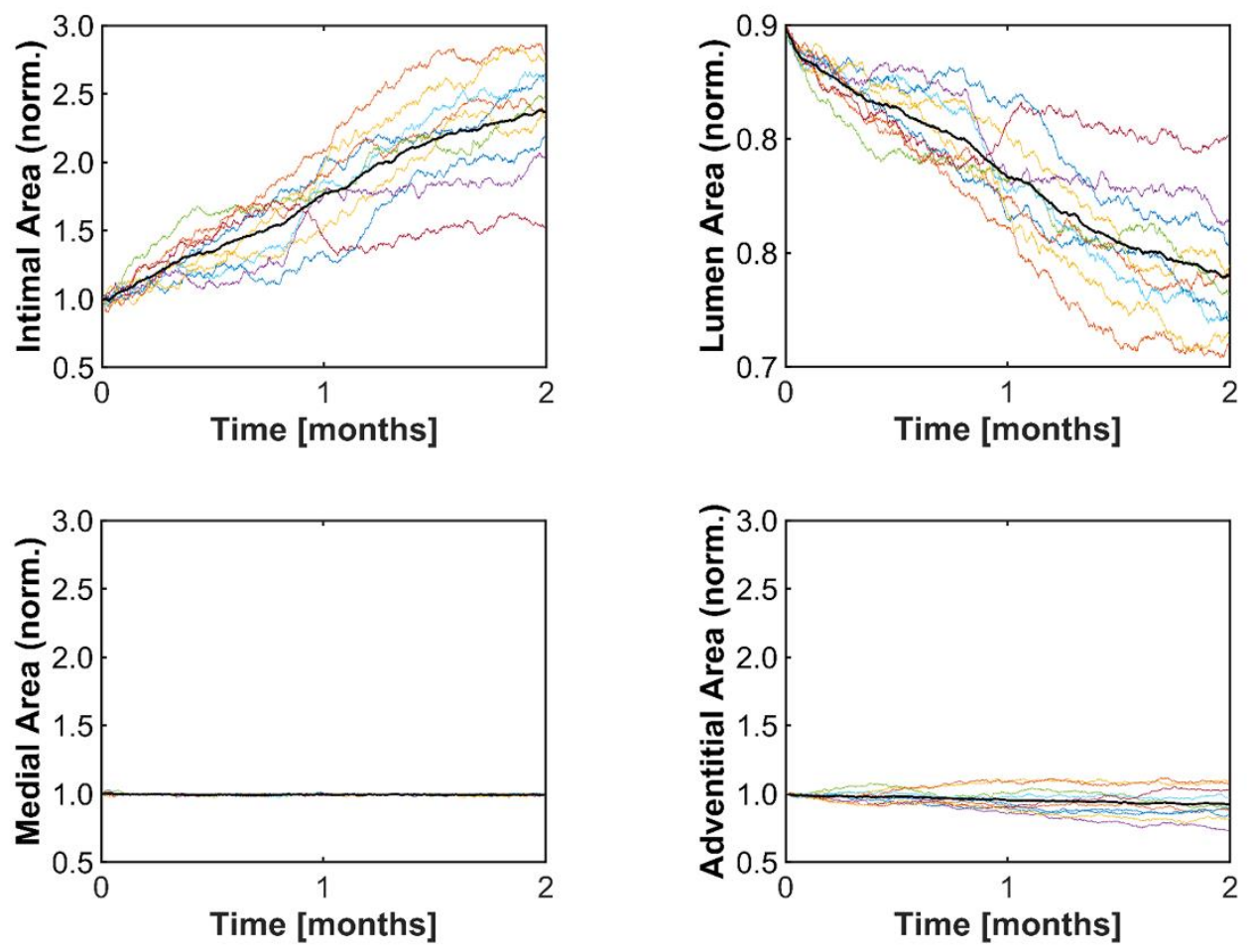

$-a-b-c-d-e-f-g-h-i-j-M e a n$

B)

Final ABM cross-sections

a.

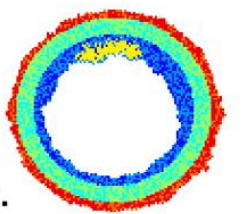

f.

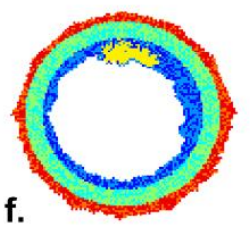

Lipid

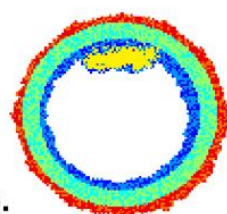

c.
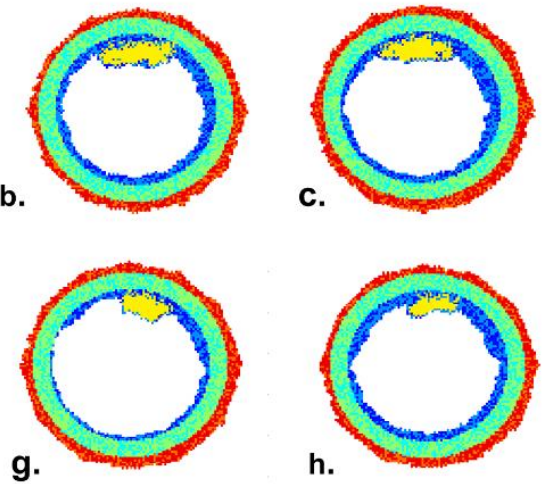

h.

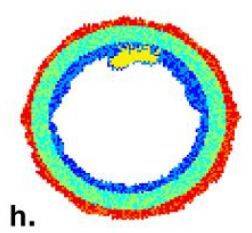

i.

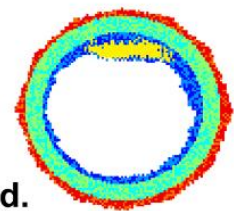

e.
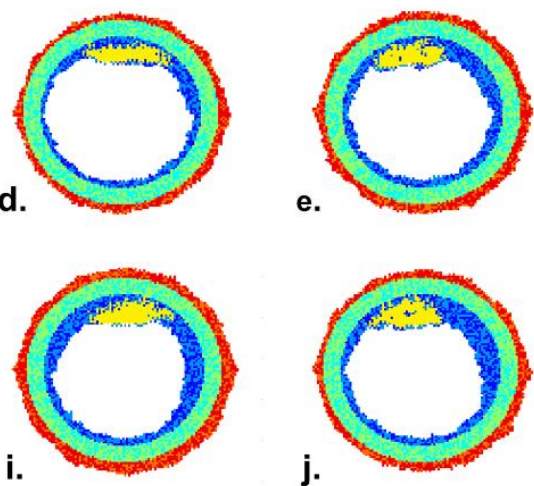

Adventitial Cell

Adventitial ECM

Fig. 9. Agent-based model (ABM) solutions for the vessel cross-sectional plane 5. A) Temporal trends of intimal, luminal, medial and adventitial areas along two simulated months, with the colored lines representing each of the ten independent simulations and the mean trend in bold black. B) Final ABM cross-sections obtained at the end of the a,...,j independent simulations. 
Finally, the severity of the replicated pathology was proportional to the degree of atherogenic character of the WSS profile. In Fig. 10, the temporal evolution of an ABM cross-section out of ten is shown for planes 4, 5 and 9, providing an example of the ABM sensitivity to WSS. The percentage of lumen wall exposed to WSS < $1 \mathrm{~Pa}$ was $0.8,16.5$ and 52.6, respectively, while the lowest recorded WSS was $0.98 \mathrm{~Pa}, 0.69 \mathrm{~Pa}$ and $0.10 \mathrm{~Pa}$. These WSS profiles triggered wall responses with different degree of intensity. Specifically, in plane 4, after two months the process of lipid infiltration was only at the beginning, with few lipid agents in the intimal layer, differently from plane 5 and 9 , where lipids started migrating into the intimal layer within the first month, leading to the generation of a well discernible lipid core. In plane 9, the pathologic wall remodeling was faster than plane 5; moreover, although after one month the lipid core did not change significantly, the intima continued to grow, also thickening the layer between the lipid core and the lumen. At day 60, the configuration of plane 9 presented a more critical scenario compared to plane 5, in which the size of the lipid core was not stabilized yet and the lumen area was still largely preserved. Coherently with the definition and classification of advanced atherosclerotic plaque proposed by Stary et. al [46], the ABM configuration of plane 5 at day 60 (same as Fig. 8B) qualitatively resembles a type IV lesion, characterized by a dense accumulation of lipids without substantial lumen area change, and might progress to a condition of type V lesion, in which ECM is the major plaque component (as Fig. 10, plane 9 at day 60).

The lumen stenosis at the end of the two months follow-up were $10 \%, 20 \%$ and $80 \%$ for plane 4,5 and 9 , respectively. 


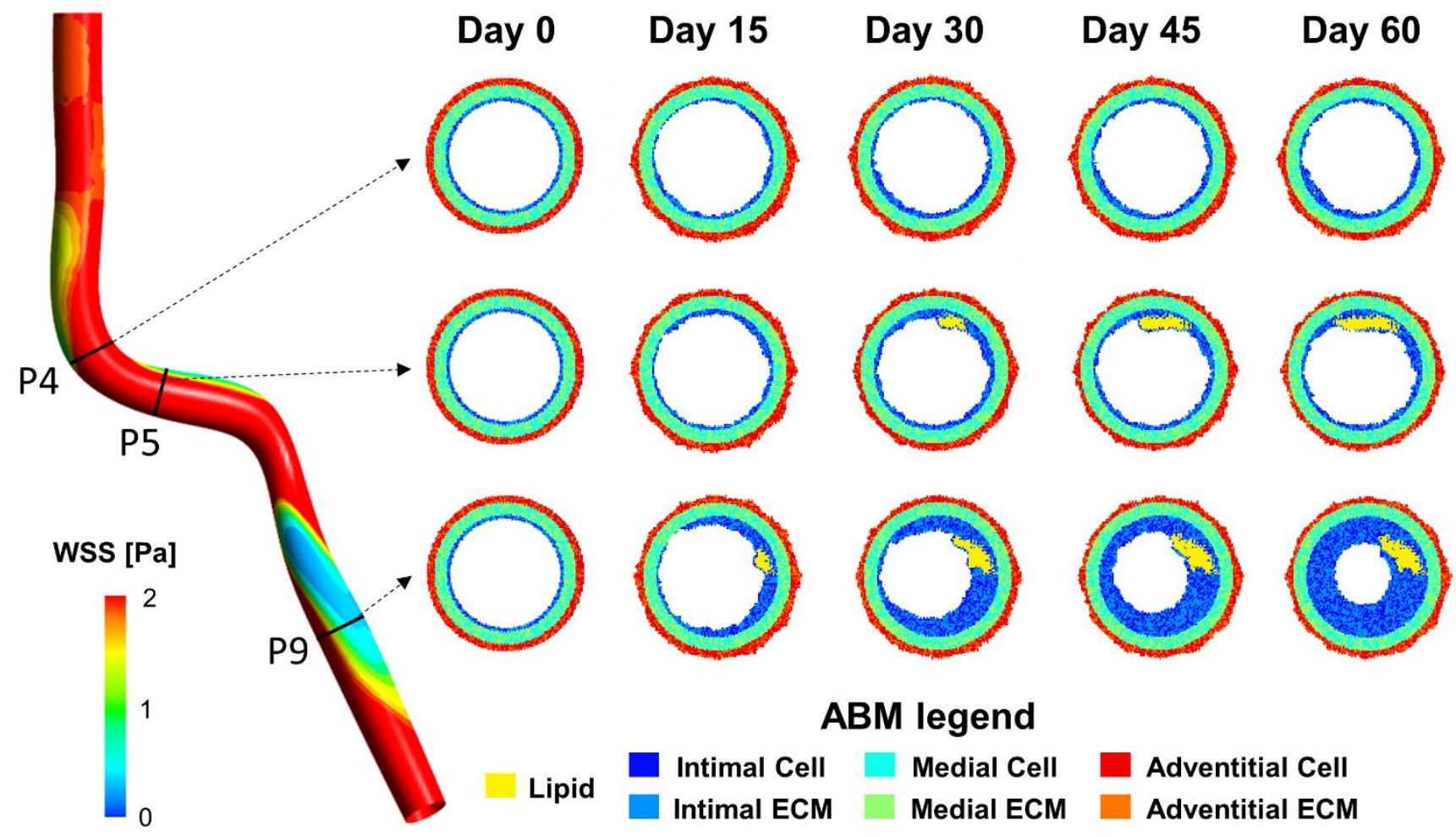

Fig. 10. Agent-based model (ABM) sensitivity to wall shear stress (WSS). On the left, the initial WSS contour, on the right, the 2-months ABM-simulated process of wall remodeling for planes 4, 5 and 9. For each plane the most representative simulation output out of ten is selected and five intermediate cross-sections are provided with a 15 days time-step.

\subsection{Sensitivity analysis}

\subsubsection{Mono-parametric sensitivity analysis}

Among the investigated parameters, the performed analysis pointed out the presence of one most influent parameter, $\alpha_{2}$, whose primary effect on the SMC and ECM dynamics propagated to the lipid dynamics and largely affected the predicted lumen area reduction. Figure 11 provides details on the model sensitivity to $\alpha_{2}$ in terms of intimal SMC (Fig. 11A), ECM (Fig. 11B), and lipids (Fig. 11C) and lumen area (Fig. 11D) showing, for each studied output, the temporal trends along the simulation and seven box plots at one month of follow-up. 
In the graphs reporting the time evolution of the variables, for each considered value of the parameter, the colored bold line represents the median trend and the associated band is the interquartile range (IQR $25^{\text {th }}-75^{\text {th }}$ percentiles). On the right, the corresponding box plots describe the data distribution of the specific variable at the end of the simulation obtained with the specific value of $\alpha_{2}$. Acting on SMC and ECM dynamics, $\alpha_{2}$ indirectly affected also lipid dynamics by anticipating or delaying the process of lipid infiltration in the intima, whose starting moment is clearly evident in Fig. $11 \mathrm{C}$ and corresponds to the point on the $\mathrm{x}$-axis at which a number of lipids greater than 0 is first observed. Specifically, the greater $\alpha_{2}$, the more SMC proliferation and ECM production were promoted, with an observed increase in such event rates and, obviously a higher intimal content of SMC and ECM at the end of the simulation (Figs. 11A-B). In turn, an augmented SMC proliferation and ECM production led to a faster thickening of the intima and, as consequence, to an earlier invasion of lipids in the wall (Fig. 11C). As expected, the starting point for lipid infiltration influenced the number of lipids in the intima observed at one month of follow-up. However, a saturation of the lipid content was observed with $\alpha_{2}=\{2.436 ; 2.854\}$, due to a control on the lipid core size introduced in the lipid dynamics algorithm. Moreover, as natural consequence of the large effect that $\alpha_{2}$ has on the agent dynamics, the lumen area was considerably influenced by such parameter (Fig. 11D). An increase in $\alpha_{2}$ enhanced lumen area reduction rate, leading to the most critical scenario (i.e. smallest final lumen area) associated with the highest $\alpha_{2}=2.854$. Significant differences among the data distributions associated with different values of $\alpha_{2}$ were detected for all the studied outputs $(\mathrm{p}<0.05)$, and details on the multiple comparisons are provided in the supplementary material (Tab. S1). Finally, the results shown by the box plots pointed out a clearly monotonic relationship between $\alpha_{2}$ and the studied outputs. 
A)

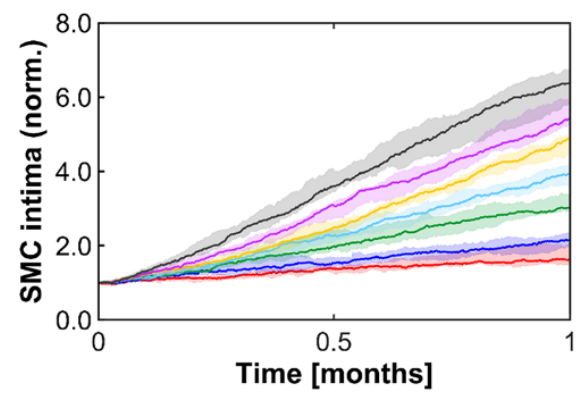

B)

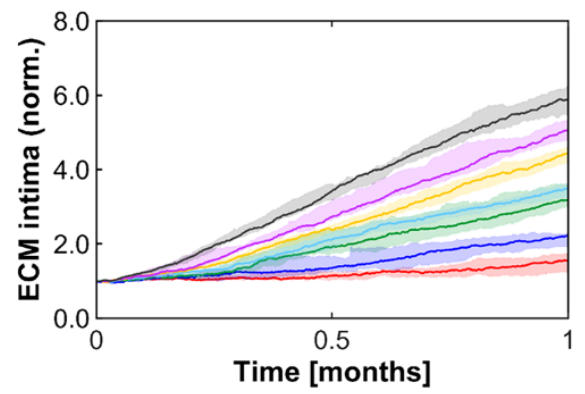

C)

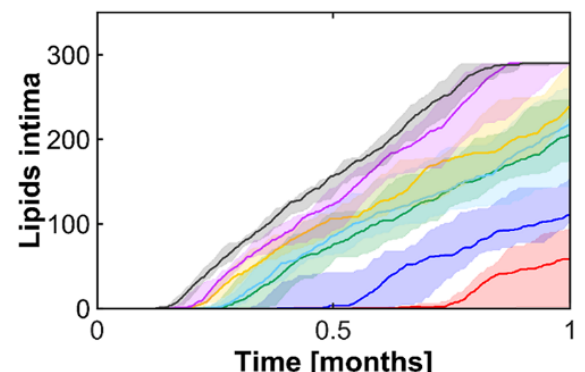

D)
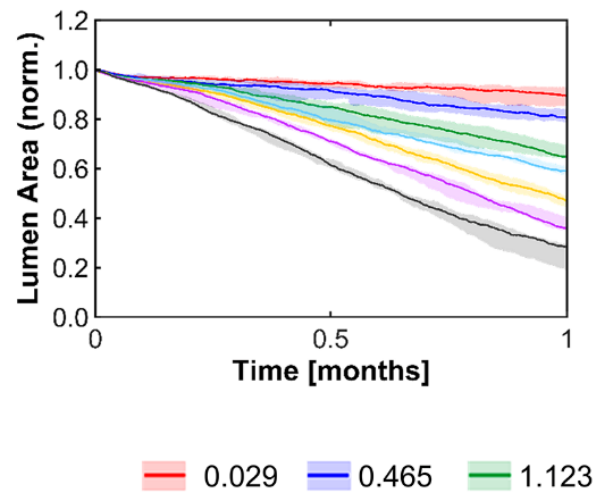
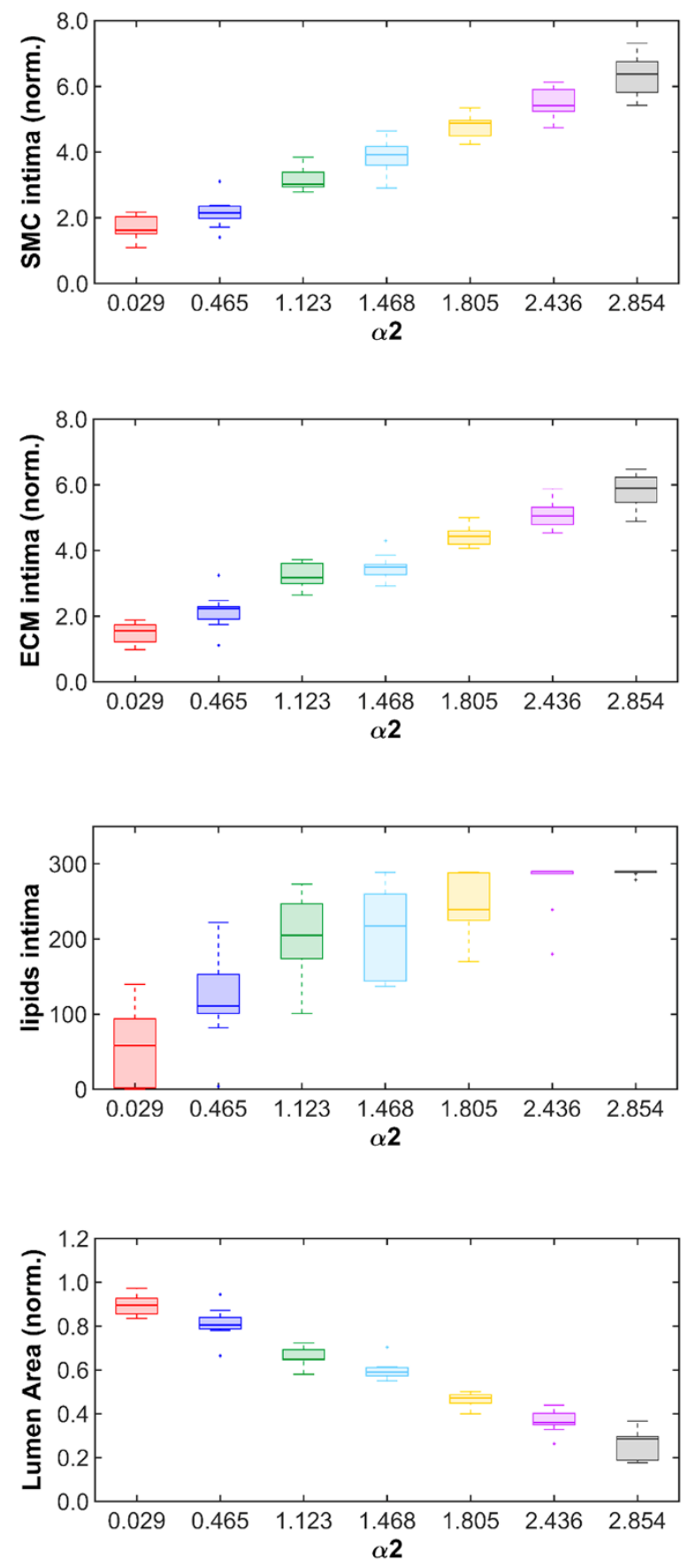

Median [IQR]

Fig. 11. Agent-based model (ABM) sensitivity to $\alpha_{2}$. ABM intimal content of (A) smooth-muscle cells (SMC), (B) extracellular matrix (ECM), (C) lipid and (D) lumen area at the variation of $\alpha_{2}$. For each target output, the temporal trends along one simulated month and box plots of the distribution at the end of the simulation are provided, with the colors corresponding to different values assumed by $\alpha_{2}$. 
The graphs of the temporal dynamics and box plots of each considered output at the variation of the parameters $\left\{\alpha_{3}, \alpha_{5}, \alpha_{6}, \alpha_{7}, I T\right.$, try $\left._{\text {lip }}\right\}$ are provided in the supplementary materials with the same modalities used for $\alpha_{2}$, see Figs. S4-9 and Tabs. S2-5. Within the studied range, parameters $\alpha_{3}$ and $\alpha_{7}$ did not show any significant influence neither in the agent dynamics, nor, as consequence, in the lumen area (Figs. S4 and S7).

While the global model output, represented by the lumen area, was affected almost exclusively by $\alpha_{2}$, which emerged as the driving parameter, the subset of parameters $\left\{\alpha_{5}, \alpha_{6}, I T\right.$, try $\left.y_{l i p}\right\}$ was identified to significantly influence lipid dynamics with minor or no effects in the other model outputs. Figure 12 provides the lipid temporal dynamics along one month of simulation and box plots of the final intimal lipid content to the variation of the parameters $\alpha_{5}$ (Fig. 12A), $\alpha_{6}$ (Fig. 12B), IT (Fig. 12C) and trylip (Fig. 12D). As regards $\alpha_{5}$, while in the range between 0.039 and 0.105 , no significant differences in the final lipid content were detected, $\alpha_{5}$ significantly affected that output when decreased below 0.039, with almost inhibition of the lipid intimal infiltration for $\alpha_{5}=0.001$. The effect of $\alpha_{5}$ on the lipid dynamics slightly propagated to the ECM dynamics, producing a reduced final ECM content in the intima for $\alpha_{5}=0.016$ and $\alpha_{5}=0.001$ ( $\left.\mathrm{p}<0.05\right)$ (Fig. S5 and Tab. S2). Similarly to $\alpha_{5}$, also $\alpha_{6}$ had an effect on the lipid dynamics only when decreased to 0.245 , although in this case a slight reduction of the lipid infiltration rate was produced with a minor consequence to the final lipid content, compared to $\alpha_{5}$ (Fig. S6 and Tab. S3). Furthermore, as expected, IT largely affected the final number of lipids, by controlling the starting point of the infiltration process. However, no propagation to the other dynamics was observed, except for a single significant difference recorded in the final content of ECM between $I T=3.728$ and $I T=16.85$, as shown in Fig. S8 and Tab. S4. Additionally, the number of chances for a lipid to invade the intima, trylip, acted on the infiltration rate, by increasing the probability that at each time step a lipid successfully enters. As for $I T$, only a significant difference was recorded in the final 
SMC content, between try $_{l i p}=3$ and try $_{l i p}=20$ (Fig. S9 and Tab. S5). As previously mentioned for $\alpha_{2}$, a saturation of the lipid content may occur due to a control on the maximum number of lipids.

Finally, none of these parameters had an influence on the lumen area, meaning that lumen area reduction is mostly due to an augmented SMC proliferation and ECM production, rather than lipid accumulation in the intima. 

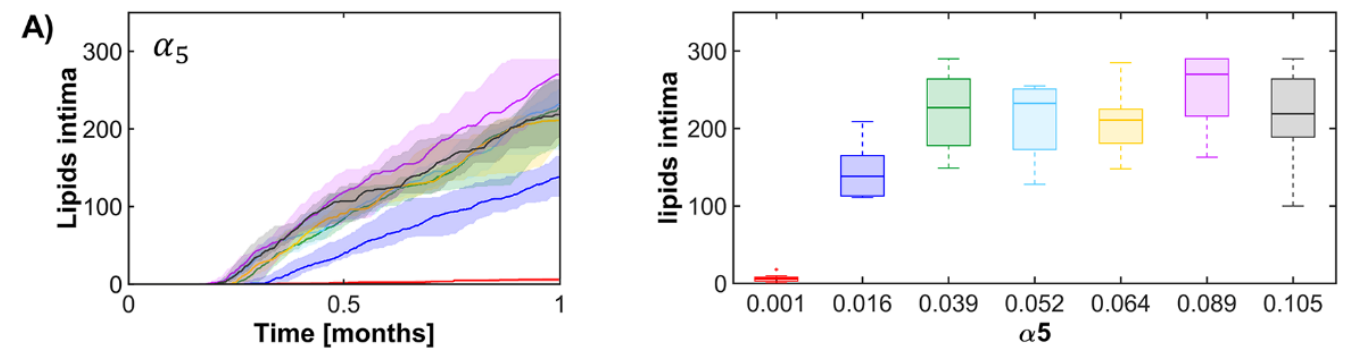

Median [IQR]

$=0.001-0.016=0.039-0.052-0.064-0.089-0.105$
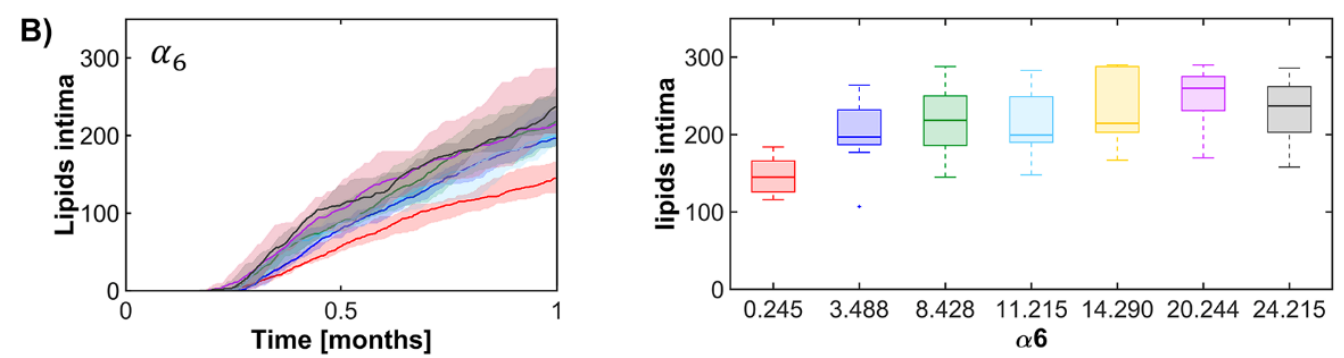

Median [IQR]

$-0.245-3.488-8.428-11.215-14.290-20.244-24.215$

\section{C)}
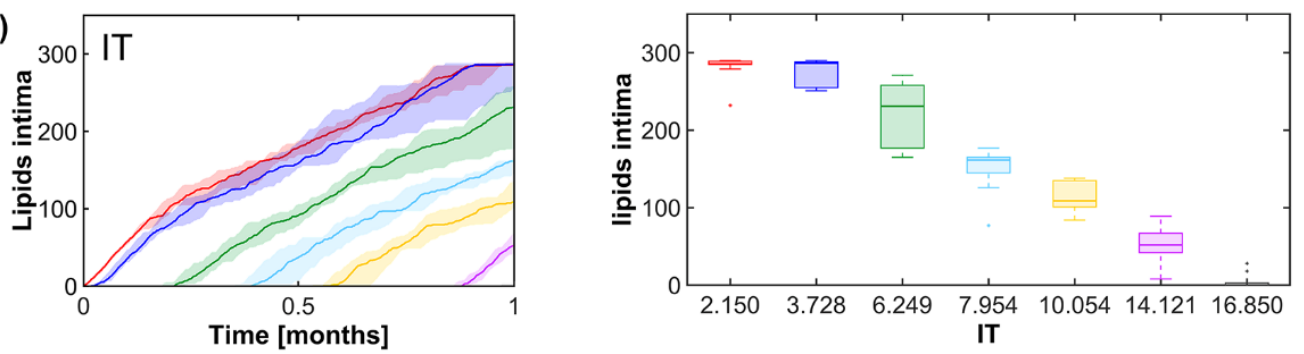

Median [IQR]

$-2.150-3.728-6.249-7.954-10.054=14.121-16.850$
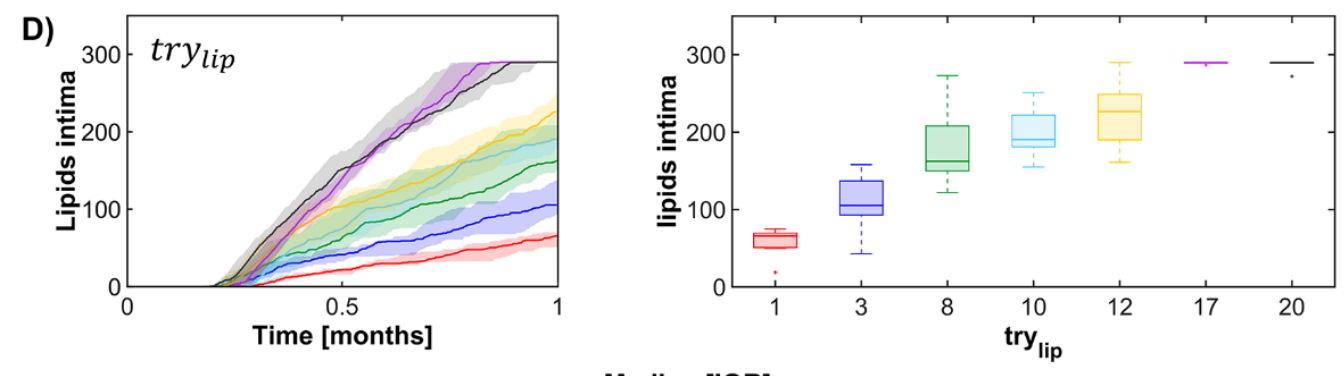

$$
\begin{array}{llllll}
-1 & -3 & -8 & -10 & -12 & -17 \quad-20
\end{array}
$$

Fig. 12. Lipid dynamics sensitivity to agent-based model (ABM) parameters. ABM intimal content of lipid at the variation of $\alpha_{5}(\mathrm{~A}), \alpha_{6}(\mathrm{~B}), I T(\mathrm{C})$ and $\operatorname{try}_{l i p}(\mathrm{D})$. For each parameter the temporal trend of lipids along one simulated month and box plots of the distribution at the end of the simulation are provided, with the colors corresponding to different values assumed by the parameter. 


\subsubsection{Multi-parametric sensitivity analysis}

Figure 13 shows the PRCCs between the target model outputs and each input parameters $\left\{\alpha_{2}, \alpha_{3}, \alpha_{5}\right.$, $\alpha_{6}, \alpha_{7}, I T$, try $\left._{l i p}\right\}$. Coherently with the mono-parametric sensitivity analysis, the final intimal content of SMC, ECM and lipids and the final lumen area were the investigated outputs. Although only values of PRCC associated with a $\mathrm{p}<0.05$ were considered as statistically significant, also PRCC values with $\mathrm{p} \approx 0.06$ were taken into account as weakly significant. In accordance with the previous analysis, $\alpha_{2}$ was identified as the most influencing parameter with significant highly positive correlations with the final amount of ECM and lipids in the intima and a significant highly negative correlation with the lumen area $(\mathrm{p}<0.05) . \alpha_{2}$ highly correlated also with the final content of SMC, but associated with a weak significance $(\mathrm{p}=0.068)$. Moreover, the remaining parameters were not found to significantly correlate neither with SMC and ECM intimal content, nor with the final lumen area, although a slight influence was recorded in some cases by the mono-parametric analysis. Finally, high correlations with weak and high significance were detected between $\alpha_{6}, I T$, try $y_{l i p}$ and the final content of lipids, with $\alpha_{6}$ and try lip $_{\text {p }}$ exhibiting positive correlations (weakly and highly significant, respectively), while IT a negative weakly significant one. 
A)

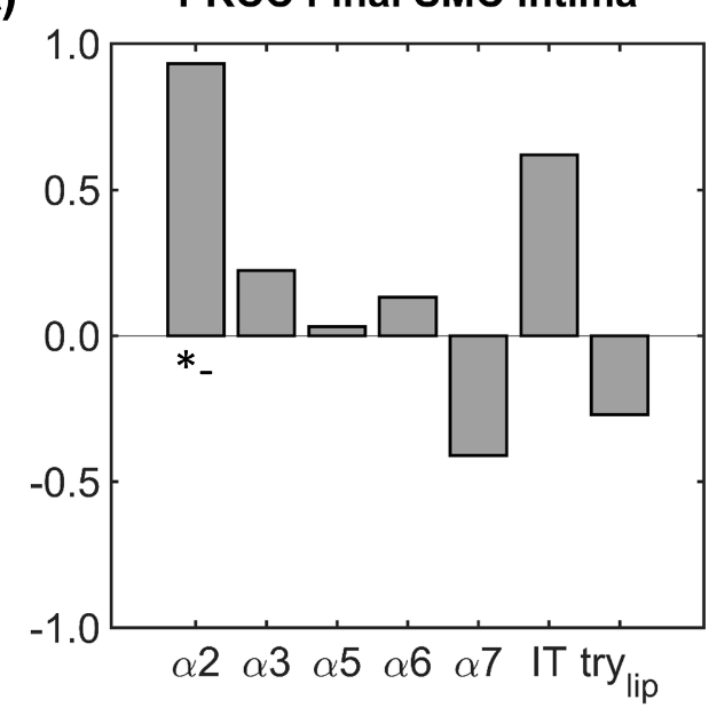

C)

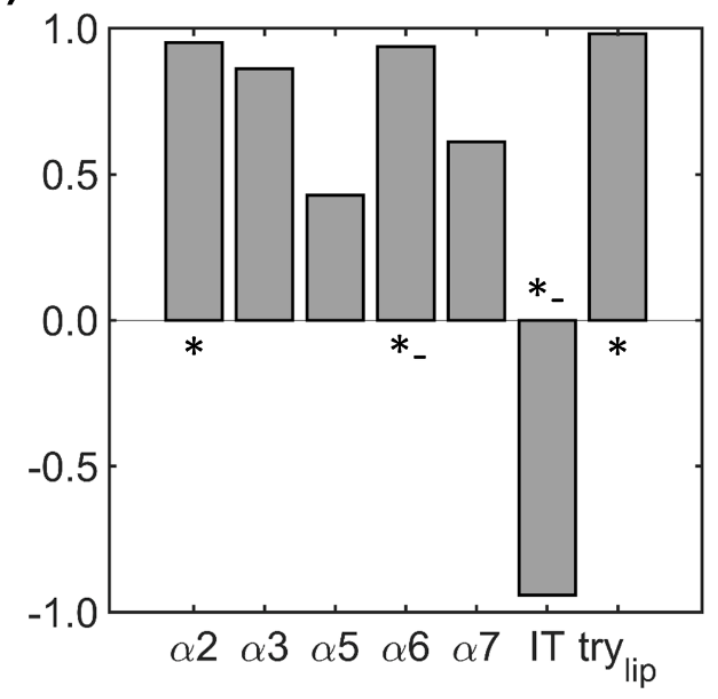

B)

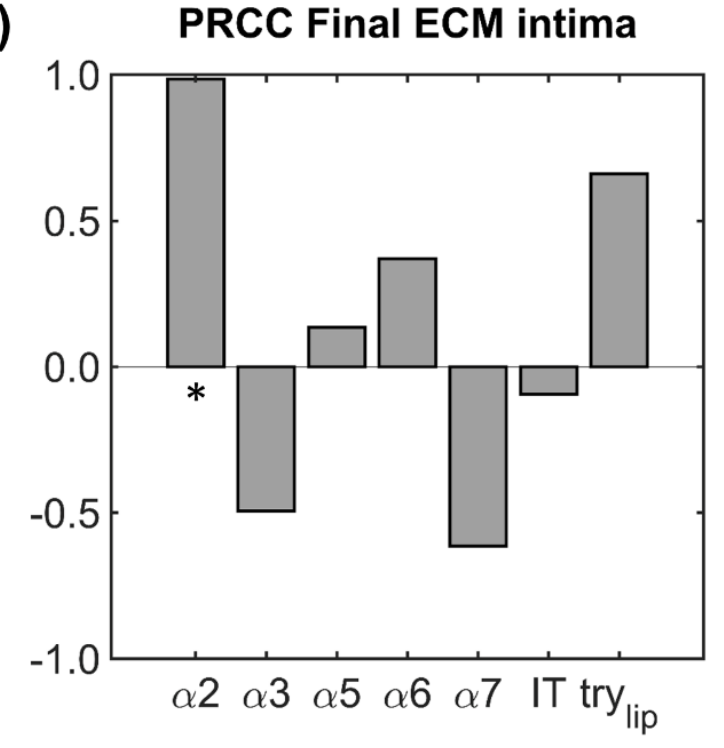

D)

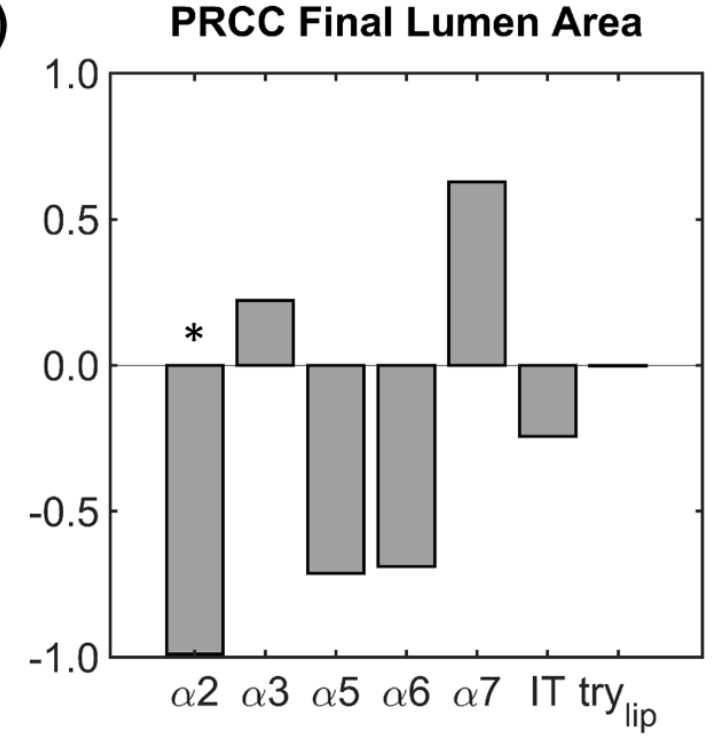

Fig. 13. Main results of the multi-parametric sensitivity analysis. Partial Rank Correlation Coefficients (PRCC) of the analyzed agent-based model (ABM) parameters and the final intimal content of (A) smooth-muscle cells (SMC), (B) extracellular matrix (ECM), (C) lipids and (D) the final lumen area. Significant PRCC are indicated with $(*)$ for $\mathrm{p}<0.05$ and $\left(*_{-}\right)$for $\mathrm{p} \approx 0.06$. 


\subsubsection{CFD-ABM coupling period}

As regards the temporal trend of the lumen area along the total 14 days of simulation, no differences resulted from the choice of a smaller or larger coupling period. Indeed, for each plane, the standard deviations of the curves obtained with the three case studies were connected or partially overlapped, meaning that the error committed by adopting the greatest coupling time (i.e. 7 days) was in the range of the noise of the stochastic ABM simulations. However, two different scenarios emerged in terms of influence of the coupling period on the ABM simulation mode, which can be physiologic or pathologic depending on the WSS profile computed at each coupling step. Indeed, while for planes where the minimum WSS values were far from $1 \mathrm{~Pa}$, there was complete agreement in the ABM simulation mode along the 14 days (Fig. 14A), discordance was observed in planes where the lowest WSS values were close to $1 \mathrm{~Pa}$ (Fig. 14B). In the last scenario, the shortest coupling period (i.e. 3.5 days) allowed catching switches between the physiologic and pathologic mode, ignored by the other two cases (see corresponding tables in Fig. 14B). Being the WSS profile of those planes very close to the physiologic/pathologic threshold, even a slight modification of the fluid-dynamic domain, obtained for example after 3.5 days of remodeling, could cause an oscillation from physiologic to pathologic condition and vice-versa. On the contrary, in the first case scenario, only a great alteration of the fluiddynamic domain could change the physiologic and atherogenic hemodynamic condition of planes 1 and 9 , respectively.

The average time of computation of the ABM was about 30 minutes per one simulated day. A total of $20,21.5$ and 20.5 hours were needed to generate the solution of the fully coupled framework at day 14, when a coupled period of 7 days, 3.5 days and 7 to 3.5 days was adopted. The greater the number of performed coupling steps, the higher the time consumption, due to the repetition of the CFD model preparation and subsequent CFD simulation. 
A)

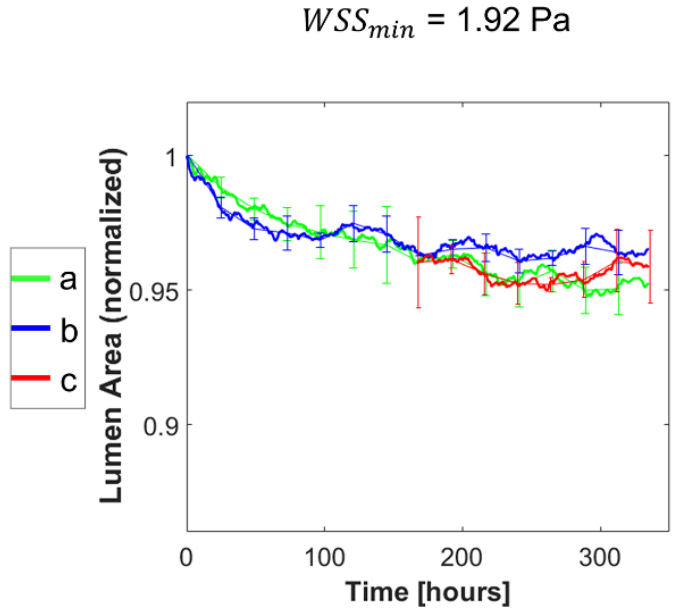

\begin{tabular}{|c|c|l|l|l|}
\cline { 2 - 5 } \multicolumn{1}{c|}{} & Day 0-3.5 & Day 3.5-7 & Day 7-10.5 & Day 10.5-14 \\
\hline a & \multicolumn{2}{|c|}{ Physiologic } & \multicolumn{2}{c|}{ Physiologic } \\
\hline b & Physiologic & Physiologic & Physiologic & Physiologic \\
\hline c & \multicolumn{2}{|c|}{ Physiologic } & Physiologic & Physiologic \\
\hline
\end{tabular}

B)

Plane 4

$W S S_{\min }=0.98 \mathrm{~Pa}$

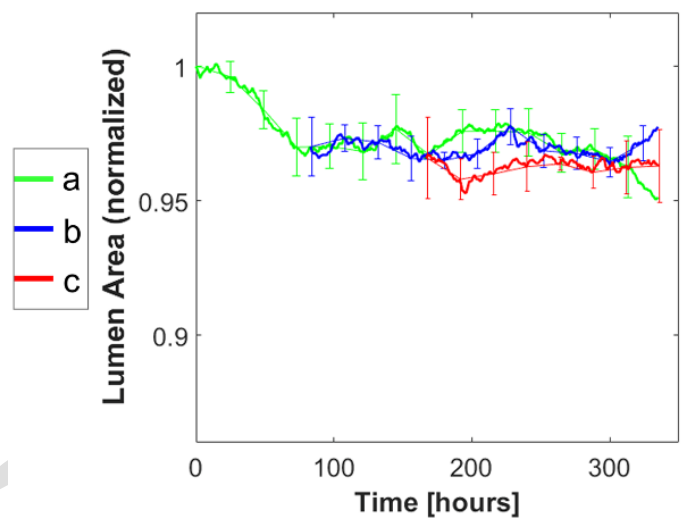

\begin{tabular}{|c|c|l|l|l|}
\cline { 2 - 5 } \multicolumn{1}{c|}{} & Day 0-3.5 & Day 3.5-7 & Day 7-10.5 & Day 10.5-14 \\
\hline a & \multicolumn{2}{|c|}{ Pathologic } & \multicolumn{2}{c|}{ Physiologic } \\
\hline b & Pathologic & Physiologic & Pathologic & Physiologic \\
\hline c & \multicolumn{2}{|c|}{ Pathologic } & Physiologic & Physiologic \\
\hline
\end{tabular}

Plane 9

$W S S_{\text {min }}=0.10 \mathrm{~Pa}$

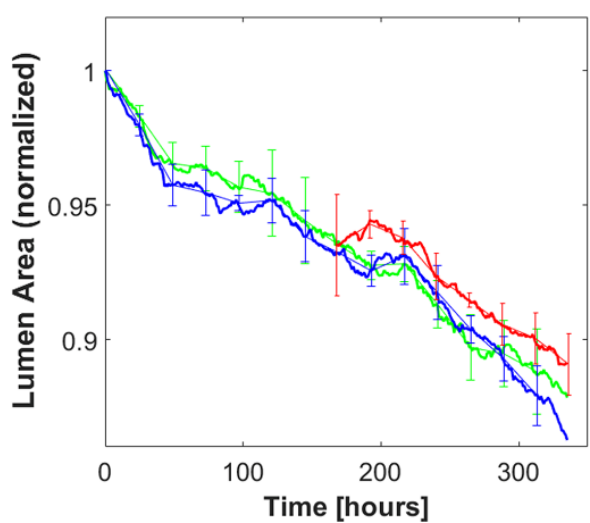

\begin{tabular}{|c|c|c|c|c|}
\cline { 2 - 5 } \multicolumn{1}{c|}{} & Day 0-3.5 & Day 3.5-7 & Day 7-10.5 & Day 10.5-14 \\
\hline a & \multicolumn{2}{|c|}{ Pathologic } & \multicolumn{2}{c|}{ Pathologic } \\
\hline b & Pathologic & Pathologic & Pathologic & Pathologic \\
\hline c & \multicolumn{2}{|c|}{ Pathologic } & Pathologic & Pathologic \\
\hline
\end{tabular}

\section{Plane 10}

$W S S_{\min }=1.06 \mathrm{~Pa}$

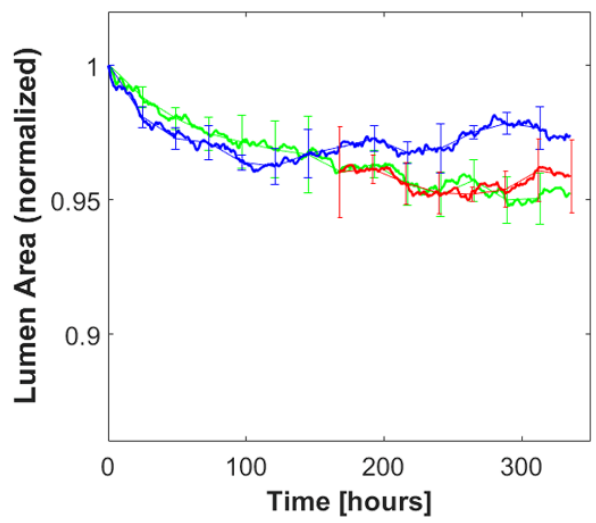

\begin{tabular}{|c|c|c|c|c|}
\cline { 2 - 5 } \multicolumn{1}{|c|}{} & Day 0-3.5 & Day 3.5-7 & Day 7-10.5 & Day 10.5-14 \\
\hline a & \multicolumn{2}{|c|}{ Physiologic } & \multicolumn{2}{c|}{ Physiologic } \\
\hline b & Physiologic & Pathologic & Pathologic & Pathologic \\
\hline c & \multicolumn{2}{|c|}{ Physiologic } & Physiologic & Physiologic \\
\hline
\end{tabular}

Fig. 14. Significant results of the sensitivity analysis of the coupling period. Results are shown for four exemplifying planes with $\mathrm{WSS}_{\min }$ far from $1 \mathrm{~Pa}(\mathbf{A})$ and planes with $\mathrm{WSS}_{\min }$ close to $1 \mathrm{~Pa}(\mathbf{B})$, corresponding to two different scenarios. For each plane the output is analyzed in terms of normalized lumen area over time (curves) and simulation mode, i.e. physiologic/pathologic, along the 14 days of follow-up, for each case study a) coupling every 7 days b) coupling every 3.5 days and c) first coupling after 7 days and then every 3.5 days. 


\section{Discussion}

Several computational studies have already used ABMs to simulate vascular adaptation processes in response to the alteration of the baseline working conditions $[16,17,26,27,34,18-25]$. In particular, in [16-24] ABMs of the arterial wall remodeling following stenting procedure were implemented to investigate the mechanisms of in-stent restenosis. However, in those works, the intervention procedure was simulated on a healthy artery, thus neglecting the underlying pathology which, instead, we consider to have a role in the final outcome. Herein we presented a novel framework that simulates the process of atherosclerotic plaque development in relation to the hemodynamics by coupling 2D ABM to CFD simulations in a 3D vessel geometry. Furthermore, we performed a detailed sensitivity analysis to identify the driving coefficients of the coupled model, thus laying the foundations for a future quantitative calibration and validation based on experimental and/or clinical data, which were not addressed in the present work.

The strengths of the present framework are (i) the inclusion of important biological aspects related to the disease, i.e. cellular and extracellular dynamics, and (ii) the definition of a loop where molecular and tissue levels are strictly interconnected, and the effect of a perturbation applied to one node is directly reflected on the other ones. The framework is modular and versatile. After a proper experimental calibration and subsequent validation, it might allow the implementation of additional phenomena, such as drug therapies or intervention procedures, on a model of diseased artery. For instance, the effect of anti-proliferative or LDL-lowering drugs (e.g. statins) might be investigated, either by introducing advection-diffusion-reaction equations for those species, or by including them in the model as agents. In both cases, the current agent rules should be modified to take into account the mutual influence between their dynamics and the newly introduced factor/agent. In this context, it might be possible to study a potential plaque stabilization or regression [49]. Moreover, the model 
might serve to create a virtual population of patients with different patterns (e.g. size and location) of lipid core, degree of stenosis and, once implemented, fibrotic cap and calcifications. Indeed, although until now the model only includes SMCs, ECM and lipid agents, implementation of calcifications is currently under investigation. This, together with further improvements and an experimental validation, might allow investigating the effects of such patterns on the lesion progression and on the intervention outcomes. Finally, by using previously developed in house techniques [14,50], the framework might be also informed with monocyte related gene expression data following a specific treatment. All these aspects are thought to represent a turning point in terms of ability to predict the treatment outcome.

To the best of our knowledge, the only work that partially resembles the proposed framework is that by Bhui et al. [27] in which a 3D ABM was coupled to CFD simulations to simulate the WSS-driven leukocyte trans-endothelial migration and subsequent plaque formation. However, differently from their model, in our ABM plaque volumetric growth was mainly due to SMCs proliferation, ECM production and lipid accumulation (Figs. 8B, 9B and 10), rather than leukocytes infiltration. This is in accordance with the study of Doran et al. [51], in which a key role to SMCs was recognized, and, the research of Stary et al. [46], who identified ECM as the major extracellular component of fibroatheromas after lipids. Finally, while in the model of Bhui et al. [27] wall remodeling was simulated according to Glagov's phenomenon [52], in the present work, for simplicity, only the stenotic effect of plaque growth was considered. Consequently, a monotonic trend of lumen area reduction was observed in our model (Fig. 9A), while, according to [52], a compensatory enlargement of the vessel wall should preserve lumen area in the early stages of plaque formation.

Although being able to capture some major aspects of the pathologic wall remodeling associated with atherosclerosis, as shown in Figs. 8, 9 and 10, the present ABM does not replicate the formation of a fibrotic cap. However, instead of fibrous tissue, it simulates a thickening of the intima (e.g. increased SMCs and ECM) between the lipid core and the lumen in advanced stages of the plaque, as in Fig. 10. 
In view of a future implementation of structural (i.e. mechanical) aspects, it might be important to distinguish the fibrotic cap, since it is a key factor in determining plaque stability/instability [3]. Finally, the ABM successfully captured wall response to different WSS stimuli (Fig. 10). Although endothelial cells were not included in the ABM, the contribution of the WSS on the endothelial dysfunction, subsequently triggering the process of plaque formation, was considered through the implementation of a diffusion equation for the inflammation. Similarly, in previous works aimed at investigating the phenomenon of in-stent restenosis $[18,19]$, the endothelium was not explicitly modeled, but a method to estimate the nitric oxide was used, thus considering its influence in SMCs activity and the effect WSS exerts on it.

The sensitivity analysis performed on the ABM provided insights in the working mechanisms of the model, by identifying the most influencing parameters, the interactions among the agent dynamics and the contribution of SMC, ECM and lipid dynamics in the lumen area change. Both the monoparametric and multi-parametric sensitivity analyses recognized $\alpha_{2}$ (i.e. weight of the effect of inflammation in cell/ECM dynamics) as the driving parameter. From a designing perspective, this is the most important finding, suggesting that a future calibration of $\alpha_{2}$ will reduce most of the epistemic uncertainty associated with the model, thus improving the accuracy of the results. Moreover, in a general view, knowing in advance which is the most important parameter to be calibrated allows planning experiments optimized for the specific purpose, thus avoiding a waste of time and resources.

The reasons why $\alpha_{3}$ did not show any contribution probably lie in the considered range and in the fact that the term $\left(1+\alpha_{3} n_{\text {lip }}\right)$ only involves a minor portion of SMC/ECM agents, thus constituting a local factor that does not produce a net effect in the cell/ECM dynamics. As regards the lipid dynamics and its driving parameters, the performed sensitivity analysis pointed out some crucial aspects, previously unknown. First, the saturation of the lipid infiltration rate for $\alpha_{5}>0.039$ and $\alpha_{6}>3.488$ while keeping all the other parameter fixed, is due to the fact that, with $t_{r} y_{l i p}=10$ chances, the probability is 
already enough to allow a lipid to enter at each time step. As a consequence, if only one parameter at time is varied, trylip is the one that mostly controls the rate of lipid accumulation in the intima. This parameter may represent the global endothelium permeability to lipids. Indeed, while the probability of a single endothelial site to allow a lipid to enter the wall is computed as expressed by Eq. 8 , the number of sites at each time step potentially favorable for lipid entry constitutes a global measure that largely control the phenomenon. However, when the combined effects of the parameters were investigated, also the contribution of $\alpha_{6}$ was identified, confirming what previously stated and recognizing the potentialities of multi-parametric sensitivity analysis.

Due to the high computational cost of the ABM (mean computation time of 25.87 hours for a 2months simulation) the LHS/PRCC sensitivity analysis was performed on a small sample size and results were obtained by running $100 \mathrm{ABM}$ simulations. Consequently, only few correlations were identified as statistically significant. However, we decided to take into account also high PRCC associated with $\mathrm{p} \approx 0.06$ because we attribute the inability to get more significance for those high correlations to the small sample size. Indeed, such correlations corresponded to parameters that were found to significantly affect that specific output in the mono-parametric sensitivity analysis. On the contrary, high p-values were associated with low PRCC values and results of the mono-parametric analysis revealing no relationship between said input and output. A low efficacy of the PRCC is, indeed, associated with non-monotonic input-output relationships [45].

To obtain more reliable correlation estimates between each output and input, it is necessary to hugely increase the sample size, implying large computational effort. For this purpose, the Matlab code of the ABM might be converted in $\mathrm{C}$, which is thought to extremely reduce the computation time for each ABM simulation. This will also allow extending the sensitivity analysis to more input parameters that were not considered in the present work, namely those related to the diffusion equation for the inflammation and the threshold on the WSS condition. In particular, the WSS threshold of $1 \mathrm{~Pa}$ is a 
strong assumption which is thought to largely affect the output of the model. In fact, in case the threshold was lowered to $0.5 \mathrm{~Pa}$, for example, plane 5 in Fig. 10, whose minimum WSS is $0.69 \mathrm{~Pa}$, would remain in physiologic condition as plane 1, and plane 9 would develop a less severe plaque. Moreover, in a future perspective, the combination of sensitivity analysis and inverse problem solution proposed by Casarin et al. $[53,54]$ can be a valuable tool to further narrow the range of optimal setting of the ABM model coefficients.

The sensitivity analysis on the coupling period revealed that a shorter coupling time should be preferred until the ABM simulation mode for each plane stabilizes, namely until the WSS profile become clearly pathologic/physiologic (i.e. far from $1 \mathrm{~Pa}$ ) or switching behaviors have not been detected for enough time. However, in this work only three cases of coupling period were investigated. The automation of the fully coupled CFD-ABM framework will reduce the user time consumption in the coupling processes, namely for the generation of the CFD model, the CFD simulation settings and the initialization of the subsequent ABM simulations. Once automated, a more extensive sensitivity analysis on the coupling period will be possible, as well as a sensitivity analysis on the number $M$ of 2D cross-sectional planes, which was not addressed in the present work, although considered important to determine the effect of the spatial resolution on the results. Finally, the automation of the framework and the conversion of the Matlab code in $\mathrm{C}$, will facilitate the calibration of the ABM parameters on patient-specific geometries and the future validation, which otherwise would require excessive computational efforts and time.

\section{Conclusions}

In this methodological work, we developed a multiscale CFD-ABM framework, able to capture the mutual interaction between hemodynamics and arterial wall remodeling in atherosclerosis. The 
framework successfully simulated plaque formation in areas affected by disturbed hemodynamics of an idealized SFA model and updated the fluid dynamics following plaque growth.

Qualitatively, the ABM replicated the main morphological and compositional changes involved in atherosclerosis, generating a pathologic arterial wall configuration coherent with histological images. Quantitatively, the output of the model was associated with uncertainty, which was related to its stochasticity and the input parameters. Replicating the ABM simulations $N=10$ times keeping all the parameters fixed allowed having an estimation of the aleatory uncertainty while the combination of mono-parametric and multi-parametric sensitivity analyses provided an estimation of the output oscillation due to uncertainty in the input parameters.

The sensitivity analysis of the ABM parameters revealed that the lumen area reduction, which is the most clinically relevant effect of plaque formation, was exclusively governed by the weight of the WSS-induced inflammation, represented by $\alpha_{2}$, which acts on the SMC proliferation and ECM production in the intima layer. As a consequence, $\alpha_{2}$ was responsible for most of the uncertainty of the model output. This finding suggests that the identification of the exact value for $\alpha_{2}$ will be a turning point towards the definition of a simplified, but reliable model. Other parameters were found to influence the process of formation of the lipid core, without affecting neither SMC/ECM dynamics, nor the lumen area change. These parameters, having a local effect, are less important to be calibrated, but may express the inter-variability of the lipid core size among individuals.

In conclusion, the results of the sensitivity analysis lay the foundations for a future parameter calibration and model validation based on experimental and/or clinical data, which is required for a more systematic assessment of the reliability and usability of the present multiscale CFD-ABM framework of atherosclerosis. 


\section{Acknoledgements}

A. Corti, C. Chiastra and M. Colombo have been supported by Fondazione Cariplo, Italy (Grant number 2017-0792, TIME). S. Casarin and M. Garbey have been supported by National Institutes of Health (NIH) grant U01HL119178-01.

\section{Conflict of interest}

The authors declare no conflicts of interest.

\section{References}

[1] A.M. Kakkar, J.D. Abbott, Percutaneous Versus Surgical Management of Lower Extremity Peripheral Artery Disease, Curr. Atheroscler. Rep. 17 (2015) 2. doi:10.1007/s11883-014-0479-0.

[2] E.R. Mohler III, Peripheral Arterial Disease: Identification and Implications, JAMA Intern. Med. 163 (2003) 2306-2314. doi:10.1001/archinte.163.19.2306.

[3] J.F. Bentzon, F. Otsuka, R. Virmani, E. Falk, Mechanisms of Plaque Formation and Rupture, Circ. Res. 114 (2014) 1852-1866. doi:10.1161/CIRCRESAHA.114.302721.

[4] Y.S. Chatzizisis, A.U. Coskun, M. Jonas, E.R. Edelman, C.L. Feldman, P.H. Stone, Role of Endothelial Shear Stress in the Natural History of Coronary Atherosclerosis and Vascular Remodeling, J. Am. Coll. Cardiol. 49 (2007) 2379-2393. doi:10.1016/j.jacc.2007.02.059.

[5] M.B. Malas, N. Enwerem, U. Qazi, B. Brown, E.B. Schneider, T. Reifsnyder, J.A. Freischlag, B.A. Perler, Comparison of surgical bypass with angioplasty and stenting of superficial femoral artery disease, J. Vasc. Surg. 59 (2014) 129-135. doi:10.1016/j.jvs.2013.05.100. 
[6] M. Soufi, A.M. Sattler, B. Maisch, J.R. Schaefer, Molecular Mechanisms Involved in Atherosclerosis, Herz. 27 (2002) 637-648. doi:10.1007/s00059-002-2431-2.

[7] D.K. Arnett, A.E. Baird, R.A. Barkley, C.T. Basson, E. Boerwinkle, S.K. Ganesh, D.M.

Herrington, Y. Hong, C. Jaquish, D.A. McDermott, C.J. O’Donnell, Relevance of Genetics and Genomics for Prevention and Treatment of Cardiovascular Disease, Circulation. 115 (2007) 2878-2901. doi:10.1161/CIRCULATIONAHA.107.183679.

[8] H.P. Fischer, D. Ph, Mathematical Modeling of Complex Biological Systems, Birkhäuser Boston, Boston, MA, 2006. doi:10.1007/978-0-8176-4503-8.

[9] R.L. Winslow, N. Trayanova, D. Geman, M.I. Miller, Computational Medicine: Translating Models to Clinical Care, Sci. Transl. Med. 4 (2012) 158rv11-158rv11. doi:10.1126/scitranslmed.3003528.

[10] S. Morlacchi, F. Migliavacca, Modeling Stented Coronary Arteries: Where We are, Where to Go, Ann. Biomed. Eng. 41 (2013) 1428-1444. doi:10.1007/s10439-012-0681-6.

[11] A.P. Antoniadis, P. Mortier, G. Kassab, G. Dubini, N. Foin, Y. Murasato, A.A. Giannopoulos, S. Tu, K. Iwasaki, Y. Hikichi, F. Migliavacca, C. Chiastra, J.J. Wentzel, F. Gijsen, J.H.C. Reiber, P. Barlis, P.W. Serruys, D.L. Bhatt, G. Stankovic, E.R. Edelman, G.D. Giannoglou, Y. Louvard, Y.S. Chatzizisis, Biomechanical Modeling to Improve Coronary Artery Bifurcation Stenting, JACC Cardiovasc. Interv. 8 (2015) 1281-1296. doi:10.1016/j.jcin.2015.06.015.

[12] P.D. Morris, A. Narracott, H. von Tengg-Kobligk, D.A. Silva Soto, S. Hsiao, A. Lungu, P. Evans, N.W. Bressloff, P. V. Lawford, D.R. Hose, J.P. Gunn, Computational fluid dynamics modelling in cardiovascular medicine, Heart. 102 (2016) 18-28. doi:10.1136/heartjnl-2015308044.

[13] M. Garbey, S.A. Berceli, A dynamical system that describes vein graft adaptation and failure, J. Theor. Biol. 336 (2013) 209-220. doi:10.1016/j.jtbi.2013.07.006. 
[14] S. Casarin, S.A. Berceli, M. Garbey, Linking Gene Dynamics to Intimal Hyperplasia - A Predictive Model of Vein Graft Adaptation, Procedia Comput. Sci. 108 (2017) 1842-1851. doi:10.1016/j.procs.2017.05.053.

[15] F. Alfonso, R.A. Byrne, F. Rivero, A. Kastrati, Current Treatment of In-Stent Restenosis, J. Am. Coll. Cardiol. 63 (2014) 2659-2673. doi:10.1016/j.jacc.2014.02.545.

[16] A. Caiazzo, D. Evans, J.-L. Falcone, J. Hegewald, E. Lorenz, B. Stahl, D. Wang, J. Bernsdorf, B. Chopard, J. Gunn, R. Hose, M. Krafczyk, P. Lawford, R. Smallwood, D. Walker, A. Hoekstra, A Complex Automata approach for in-stent restenosis: Two-dimensional multiscale modelling and simulations, J. Comput. Sci. 2 (2011) 9-17. doi:10.1016/j.jocs.2010.09.002.

[17] H. Tahir, A.G. Hoekstra, E. Lorenz, P. V. Lawford, D.R. Hose, J. Gunn, D.J.W. Evans, Multiscale simulations of the dynamics of in-stent restenosis: impact of stent deployment and design, Interface Focus. 1 (2011) 365-373. doi:10.1098/rsfs.2010.0024.

[18] H. Tahir, C. Bona-Casas, A.G. Hoekstra, Modelling the Effect of a Functional Endothelium on the Development of In-Stent Restenosis, PLoS One. 8 (2013) e66138. doi:10.1371/journal.pone.0066138.

[19] P.S. Zun, T. Anikina, A. Svitenkov, A.G. Hoekstra, A Comparison of Fully-Coupled 3D In-Stent Restenosis Simulations to In-vivo Data, Front. Physiol. 8 (2017) 1-12. doi:10.3389/fphys.2017.00284.

[20] C.J. Boyle, A.B. Lennon, M. Early, D.J. Kelly, C. Lally, P.J. Prendergast, Computational simulation methodologies for mechanobiological modelling: a cell-centred approach to neointima development in stents, Philos. Trans. R. Soc. A Math. Phys. Eng. Sci. 368 (2010) 2919-2935. doi:10.1098/rsta.2010.0071.

[21] C.J. Boyle, In Silico Prediction of the Mechanobiological Response of Arterial Tissue: Application to Angioplasty and Stenting, J. Biomech. Eng. 133 (2011) 081001. 
doi:10.1115/1.4004492.

[22] H. Zahedmanesh, H. Van Oosterwyck, C. Lally, A multi-scale mechanobiological model of instent restenosis: deciphering the role of matrix metalloproteinase and extracellular matrix changes, Comput. Methods Biomech. Biomed. Engin. 17 (2014) 813-828. doi:10.1080/10255842.2012.716830.

[23] D.R. Nolan, C. Lally, An investigation of damage mechanisms in mechanobiological models of in-stent restenosis, J. Comput. Sci. 24 (2018) 132-142. doi:10.1016/j.jocs.2017.04.009.

[24] A.E. Curtin, L. Zhou, An Agent-Based Model of the Response to Angioplasty and Bare-Metal Stent Deployment in an Atherosclerotic Blood Vessel, PLoS One. 9 (2014) e94411. doi:10.1371/journal.pone.0094411.

[25] F. Pappalardo, S. Musumeci, S. Motta, Modeling immune system control of atherogenesis, Bioinformatics. 24 (2008) 1715-1721. doi:10.1093/bioinformatics/btn306.

[26] R.N. Poston, D.R.M.P. A, Typical Atherosclerotic Plaque Morphology Produced in Silico by an Atherogenesis Model Based on Self-Perpetuating Propagating Macrophage Recruitment, Math. Model. Nat. Phenom. 2 (2007) 142-149.

[27] R. Bhui, H.N. Hayenga, An agent-based model of leukocyte transendothelial migration during atherogenesis, PLoS Comput. Biol. 13 (2017) 1-23. doi:10.1371/journal.pcbi.1005523.

[28] A. Corti, S. Casarin, C. Chiastra, M. Colombo, F. Migliavacca, M. Garbey, A Multiscale Model of Atherosclerotic Plaque Development: Toward a Coupling Between an Agent-Based Model and CFD Simulations, in: Lect. Notes Comput. Sci. (Including Subser. Lect. Notes Artif. Intell. Lect. Notes Bioinformatics), 2019: pp. 410-423. doi:10.1007/978-3-030-22747-0_31.

[29] A. Saltelli, K. Aleksankina, W. Becker, P. Fennell, F. Ferretti, N. Holst, S. Li, Q. Wu, Why so many published sensitivity analyses are false: A systematic review of sensitivity analysis practices, Environ. Model. Softw. 114 (2019) 29-39. doi:10.1016/j.envsoft.2019.01.012. 
[30] M. Colombo, M. Bologna, M. Garbey, S. Berceli, Y. He, J.F. Rodriguez Matas, F. Migliavacca, C. Chiastra, Computing patient-specific hemodynamics in stented femoral artery models obtained from computed tomography using a validated 3D reconstruction method, Med. Eng. Phys. (2019). doi:10.1016/J.MEDENGPHY.2019.10.005.

[31] R. Ponzini, C. Vergara, A. Redaelli, A. Veneziani, Reliable CFD-based estimation of flow rate in haemodynamics measures, Ultrasound Med. Biol. 32 (2006) 1545-1555. doi:10.1016/j.ultrasmedbio.2006.05.022.

[32] M. Caputo, C. Chiastra, C. Cianciolo, E. Cutrì, G. Dubini, J. Gunn, B. Keller, F. Migliavacca, P. Zunino, Simulation of oxygen transfer in stented arteries and correlation with in-stent restenosis, Int. j. Numer. Method. Biomed. Eng. 29 (2013) 1373-1387. doi:10.1002/cnm.2588.

[33] C. Chiastra, F. Iannaccone, M.J. Grundeken, F.J.H. Gijsen, P. Segers, M. De Beule, P.W. Serruys, J.J. Wykrzykowska, A.F.W. van der Steen, J.J. Wentzel, Coronary fractional flow reserve measurements of a stenosed side branch: a computational study investigating the influence of the bifurcation angle, Biomed. Eng. Online. 15 (2016) 91. doi:10.1186/s12938-0160211-0.

[34] M. Garbey, S. Casarin, S.A. Berceli, Vascular Adaptation: Pattern Formation and Cross Validation between an Agent Based Model and a Dynamical System, J. Theor. Biol. 429 (2017) 149-163. doi:10.1016/j.jtbi.2017.06.013.

[35] M. Garbey, S. Casarin, S.A. Berceli, A versatile hybrid agent-based, particle and partial differential equations method to analyze vascular adaptation, Biomech. Model. Mechanobiol. 18 (2019) 29-44. doi:10.1007/s10237-018-1065-0.

[36] E.G.J. Vermeulen, H.W.M. Niessen, M. Bogels, C.D.A. Stehouwer, J.A. Rauwerda, V.W.M. van Hinsbergh, Decreased Smooth Muscle Cell/Extracellular Matrix Ratio of Media of Femoral Artery in Patients With Atherosclerosis and Hyperhomocysteinemia, Arterioscler. Thromb. 
Vasc. Biol. 21 (2001) 573-577. doi:10.1161/01.ATV.21.4.573.

[37] D.P. Sokolis, Passive mechanical properties and constitutive modeling of blood vessels in relation to microstructure, Med. Biol. Eng. Comput. 46 (2008) 1187-1199. doi:10.1007/s11517008-0362-7.

[38] D. Sindram, K. Martin, J.P. Meadows, A.S. Prabhu, J.J. Heath, I.H. McKillop, D.A. Iannitti, Collagen-elastin ratio predicts burst pressure of arterial seals created using a bipolar vessel sealing device in a porcine model, Surg. Endosc. 25 (2011) 2604-2612. doi:10.1007/s00464011-1606-4.

[39] C. Cheng, R. de Crom, R. van Haperen, F. Helderman, B.M. Gourabi, L.C.A. van Damme, S.W. Kirschbaum, C.J. Slager, A.F.W. van der Steen, R. Krams, The Role of Shear Stress in Atherosclerosis: Action Through Gene Expression and Inflammation?, Cell Biochem. Biophys. 41 (2004) 279-294. doi:10.1385/CBB:41:2:279.

[40] H. Samady, P. Eshtehardi, M.C. McDaniel, J. Suo, S.S. Dhawan, C. Maynard, L.H. Timmins, A.A. Quyyumi, D.P. Giddens, Coronary artery wall shear stress is associated with progression and transformation of atherosclerotic plaque and arterial remodeling in patients with coronary artery disease, Circulation. 124 (2011) 779-788. doi:10.1161/CIRCULATIONAHA.111.021824.

[41] O. Schlager, A. Giurgea, C. Margeta, D. Seidinger, S. Steiner-Boeker, B. Van Der Loo, R. Koppensteiner, Wall shear stress in the superficial femoral artery of healthy adults and its response to postural changes and exercise, Eur. J. Vasc. Endovasc. Surg. 41 (2011) 821-827. doi:10.1016/j.ejvs.2011.01.006.

[42] A.C. Doran, N. Meller, C.A. McNamara, Role of smooth muscle cells in the initiation and early progression of atherosclerosis, Arterioscler. Thromb. Vasc. Biol. (2008). doi:10.1161/ATVBAHA.107.159327. 
[43] F. Otsuka, M. Nakano, K. Sakakura, E. Ladich, F.D. Kolodgie, R. Virmani, Unique demands of the femoral anatomy and pathology and the need for unique interventions, J. Cardiovasc. Surg. (Torino). (2013).

[44] H. Ota, W. Yu, H.R. Underhill, M. Oikawa, L. Dong, X. Zhao, N.L. Polissar, B. Neradilek, T. Gao, Z. Zhang, Z. Yan, M. Guo, Z. Zhang, T.S. Hatsukami, C. Yuan, Hemorrhage and large lipid-rich necrotic cores are independently associated with thin or ruptured fibrous caps: an in vivo 3T MRI study, Arterioscler. Thromb. Vasc. Biol. 29 (2009) 1696-1701. doi:10.1161/ATVBAHA.109.192179.

[45] S. Marino, I.B. Hogue, C.J. Ray, D.E. Kirschner, A methodology for performing global uncertainty and sensitivity analysis in systems biology, J. Theor. Biol. 254 (2008) 178-196. doi:10.1016/j.jtbi.2008.04.011.

[46] H.C. Stary, A.B. Chandler, R.E. Dinsmore, V. Fuster, S. Glagov, W. Insull, M.E. Rosenfeld, C.J. Schwartz, W.D. Wagner, R.W. Wissler, A Definition of Advanced Types of Atherosclerotic Lesions and a Histological Classification of Atherosclerosis, Arterioscler. Thromb. Vasc. Biol. 15 (1995) 1512-1531. doi:10.1161/01.ATV.15.9.1512.

[47] U. Schäfer, Y. Ho, C. Frerker, D. Schewel, D. Sanchez-Quintana, J. Schofer, K. Bijuklic, F. Meincke, T. Thielsen, F. Kreidel, K.-H. Kuck, Direct Percutaneous Access Technique for Transaxillary Transcatheter Aortic Valve Implantation, JACC Cardiovasc. Interv. 5 (2012) 477486. doi:10.1016/j.jcin.2011.11.014.

[48] K.K.L. Wong, P. Thavornpattanapong, S.C.P. Cheung, Z. Sun, J. Tu, Effect of calcification on the mechanical stability of plaque based on a three-dimensional carotid bifurcation model, BMC Cardiovasc. Disord. 12 (2012) 7. doi:10.1186/1471-2261-12-7.

[49] S.O. Almeida, M. Budoff, Effect of statins on atherosclerotic plaque, Trends Cardiovasc. Med. (2019). doi:10.1016/j.tcm.2019.01.001. 
[50] S. Casarin, S.A. Berceli, M. Garbey, Linking gene dynamics to vascular hyperplasia - Toward a predictive model of vein graft adaptation, PLoS One. 12 (2017) 1-26.

doi:10.1371/journal.pone.0187606.

[51] A.C. Doran, N. Meller, C.A. McNamara, Role of smooth muscle cells in the initiation and early progression of atherosclerosis, Arterioscler. Thromb. Vasc. Biol. 28 (2008) 812-819. doi:10.1161/ATVBAHA.107.159327.

[52] S. Glagov, E. Weisenberg, C.K. Zarins, R. Stankunavicius, G.J. Kolettis, Compensatory Enlargement of Human Atherosclerotic Coronary Arteries, N. Engl. J. Med. 316 (1987) 13711375. doi:10.1056/NEJM198705283162204.

[53] S. Casarin, S.A. Berceli, M. Garbey, A twofold usage of an agent-based model of vascular adaptation to design clinical experiments, J. Comput. Sci. 29 (2018) 59-69. doi:10.1016/j.jocs.2018.09.013.

[54] S. Casarin, S.A. Berceli, M. Garbey, A computational model-based framework to plan clinical experiments - an application to vascular adaptation biology, in: Lect. Notes Comput. Sci. (Including Subser. Lect. Notes Artif. Intell. Lect. Notes Bioinformatics), 2018: pp. 352-362. doi:10.1007/978-3-319-93698-7_27. 